\title{
SOME EXAMPLES OF REAL ALGEBRAIC AND REAL PSEUDOHOLOMORPHIC CURVES
}

\author{
S.Yu. OREVKOV
}

To Oleg Viro

\section{INTRODUCTION}

In this paper we construct several examples (series of examples) of real algebraic and real pseudoholomorphic curves in $\mathbb{R P}^{2}$ in which we tried to maximize different characteristics among curves of a given degree. In $\S 2$, this is the number of nonempty ovals; in $\S 4$, the number of ovals of the maximal depth; in $\S 5$, the number $n$ such that the curve has an $A_{n}$ singularity. In the pseudoholomorphic case, the questions of $\S 4$ and $\S 5$ are equivalent to the same problem about braids, which is studied in $\S 3$. In $\S 6.1$, we construct a real algebraic $M$-curve of degree $4 d+1$ with four nests of depth $d$ (which shows that the congruence mod 8 proven in a joint paper with Viro is "non-empty"). In $\S 6.2$, we generalize this construction. In $\S 7$, we construct real algebraic $M$-curves of degree 9 with a single exterior oval and we classify such curves up to isotopy.

Let $\alpha=\lim \sup \left(\alpha_{m} / m^{2}\right)$ where $\alpha_{m}$ is twice the maximal number $n$ such that there exists an algebraic curve in $\mathbb{C P}^{2}$ of degree $m$ with an $A_{n}$ singularity. Similarly, let $\beta=\lim \sup \left(\beta_{k} / k^{2}\right)$ where $\beta_{k}=\max l_{k-2}(A)$ where $l_{k-2}(A)$ is the number of ovals of $A$ of depth $k-1$ and the maximum is taken over all real algebraic curves in $\mathbb{R P}^{2}$ of degree $2 k$. Let $\alpha_{\mathrm{ph}}$ and $\beta_{\mathrm{ph}}$ be the same numbers for pseudoholomorphic curves. In the following table we summarize all known estimates for these numbers (LB/UB stand for lower/upper bound).

\begin{tabular}{ll}
\hline 1 & Evident LB for $\alpha, \beta, \alpha_{\mathrm{ph}}, \beta_{\mathrm{ph}}$ \\
$15 / 14,8-4 \sqrt{3}$ & LB for $\alpha$ from [14], [4] \\
$9 / 8$ & LB for $\beta$ proved in $\S 3.3$ \\
$7 / 6$ & LB for $\alpha$ proved in $\S 4$ \\
$4 / 3$ & LB for $\alpha_{\mathrm{ph}}$ and $\beta_{\mathrm{ph}}$ proved in $\S 2-4$ \\
$3 / 2$ & UB for $\alpha, \beta, \alpha_{\mathrm{ph}}, \beta_{\mathrm{ph}}$ coming from signature estimates \\
2 & Evident UB for $\alpha, \beta, \alpha_{\mathrm{ph}}, \beta_{\mathrm{ph}}$ \\
\hline
\end{tabular}




\section{ITERATION OF WIMAN'S CONSTRUCTION}

Wiman [35] proposed a method to construct real algebraic $M$-curves in $\mathbb{R P}^{2}$ which have many nests. Here we use Wiman's construction to obtain curves with many non-empty ovals. As is shown in [16], the number $I_{d}$ of isotopy types realizable by real algebraic curves of degree $d$ in $\mathbb{R P}^{2}$ has the asymptotics $\log I_{d}=C d^{2}+o\left(d^{2}\right)$ for some positive constant $C$, and the only known upper bounds for $C$ come from the fact that $C \leq \limsup f\left(L_{d} / d^{2}\right)$ where $f$ is a certain effectively computable monotone function and $L_{d}$ is the maximal number of non-empty ovals that a curve of degree $d$ may have. All known upper bounds for $L_{d}$ are of the form $d^{2} / 4+O(d)$. Here we construct real algebraic and real pseudoholomorphic curves, in particular $M$-curves, with as many non-empty ovals as we can do. The best asymptotic that we can achieve for pseudoholomorphic curves is only $d^{2} / 6+o\left(d^{2}\right)$. In the algebraic case, the obtained asymptotics are yet worse.

Let us recall Wiman's construction. We start with an $M$-curve $C$ of even degree $d$ given by an equation $F=0$. We double $C$ and then perturb it, i.e., consider a curve $C^{\prime}=\left\{F^{2}-\varepsilon G=0\right\},|\varepsilon| \ll 1$, where $G$ is some polynomial of degree $2 d$. Suppose that the curve $G=0$ meets $C$ transversally. Then each arc of $C$ where $G>0$ provides an oval of $C^{\prime}$ (obtained by doubling the arc and joining the ends). In the same way, each oval of $C$ where $G>0$ provides a pair of nested ovals of $C^{\prime}$. If we are lucky to find $G$ such that it has $2 d^{2}$ zeros on one oval of $C$ and is positive on all other ovals, then we obtain an $M$-curve which has $O\left(d^{2}\right)$ nested pairs of ovals. This can be attained, for example, if we start with an $M$-curve $C$ one of whose ovals maximally intersect a line.

When speaking of Wiman's construction, the divisor of $G$ on $C$ will be called the branching divisor.

If we work with real pseudoholomorphic curves, then we need not bother if it is possible or not to place correctly the branching divisor. Perturbing if necessarily the almost complex structure, we may place it wherever we want. The only restriction is the total degree and the parity of the number of points at each branch of $C$.

We say that an arrangement of embedded circles on $\mathbb{R P}^{2}$ is realizable by a real pseudoholomorphic curve if there exists a real pseudoholomorphic curve in $\mathbb{C P}^{2}$ whose set of real points is isotopic to the given arrangement.

Recall that a nest of depth $d$ is a union of $d$ ovals $V_{1} \cup \cdots \cup V_{d}$ such that $V_{i+1}$ is surrounded by $V_{i}, i=1, \ldots, n-1$. We say that a nest $N$ of a curve $C$ is simple if there exists an embedded disk $D \subset \mathbb{R P}^{2}$ such that $N=D \cap C$.

We shall use the encoding of isotopy types of smooth embedded curves in $\mathbb{R P}^{2}$ proposed by Viro. Namely, $n$ denotes $n$ ovals outside each other; $A \sqcup B$ denotes a union of two curves encoded by $A$ and $B$ respectively if there exist disjoint embedded disks containing them; $1\langle A\rangle$ denotes an oval surrounding a curve encoded by $A$; $n\langle A\rangle=1\langle A\rangle \sqcup \cdots \sqcup 1\langle A\rangle$ ( $n$ times).

We extend this encoding as follows. Let $1\langle\langle d\rangle\rangle$ denote a simple nest of depth $d$ and let $n\langle\langle d\rangle\rangle=1\langle\langle d\rangle\rangle \sqcup \cdots \sqcup 1\langle\langle d\rangle\rangle$ ( $n$ times). Also, if $S$ encodes the isotopy type of a curve $A$ and $A^{\prime}$ is obtained from $A$ by replacing each component by $k$ parallel copies, then we denote the isotopy type of $A^{\prime}$ by $\langle S\rangle^{k}$ or just by $S^{k}$ in the case when $S$ is of the form $n\left\langle S_{1}\right\rangle$. For, example, $2\langle\langle 3\rangle\rangle=\langle 2\rangle^{3}=2\left\langle\langle 1\rangle^{2}\right\rangle=2\langle 1\langle 1\rangle\rangle=$ $1\langle 1\langle 1\rangle\rangle \sqcup 1\langle 1\langle 1\rangle\rangle$ denotes () (?) . 
Proposition 2.1. a). For any positive integers $m$ and $k$ there exists a real pseudoholomorphic $M$-curve $C_{m, k}$ in $\mathbb{R P}^{2}$ of degree $d=2^{k} m$ realizing the isotopy type

$$
\frac{m^{2}-3 m+2}{2}\left\langle\left\langle 2^{k}\right\rangle\right\rangle \sqcup\left(\bigsqcup_{j=1}^{k-1}\left(4^{j-1} m^{2}-1\right)\left\langle\left\langle 2^{k-j}\right\rangle\right\rangle\right) \sqcup 4^{k-1} m^{2} .
$$

The number of non-empty ovals of this curve is $\frac{1}{6}\left(4^{k}-1\right) m^{2}-\frac{3}{2}\left(2^{k}-1\right) m+k=$ $\frac{1}{6}\left(d^{2}-m^{2}\right)-\frac{3}{2}(d-m)+k$. So, for each series $\left\{C_{m, k}\right\}_{k \geq 0}$ with a fixed $m$, these numbers have the asymptotics $\frac{1}{6} d^{2}+O(d)$

b). If $k \leq 3$ then, for any $m$, the $M$-curve $C_{m, k}$ can be realized algebraically. The number of non-empty ovals of $C_{m, 3}$ is $\frac{21}{2}\left(m^{2}-m\right)+3=\frac{21}{128} d^{2}+O(d)$.

c). For any $k>1$ there exists an algebraic curve $C_{2, k}^{\prime}$ of degree $d=2^{k+1}$ realizing the isotopy type

$$
3\left\langle\left\langle 2^{k-1}\right\rangle\right\rangle \sqcup\left(\bigsqcup_{j=2}^{k-1}\left(4^{j}-2^{j-2}\right)\left\langle\left\langle 2^{k-j}\right\rangle\right\rangle\right) \sqcup 4^{k}
$$

The number of ovals of $C_{2, k}^{\prime}$ is $\frac{1}{2} d^{2}-\left(\frac{k}{8}-1\right) d$, i.e., it is an $(M-r)$-curve for $r=(k-4) 2^{k-2}+2=O(d \log d)$.

The number of non-empty ovals of $C_{2, k}^{\prime}$ is $\frac{1}{6} d^{2}-\frac{k+7}{8} d+\frac{4}{3}=\frac{1}{6} d^{2}+O(d \log d)$.

Proof. All these curves are obtained by iterating Wiman's construction.

(a). We start with Harnack's curve $C_{m, 0}$ of degree $m$ and apply $k$ times Wiman's construction to it. At each step, we place the branching divisor on one empty exterior oval (see Figures 1.0 - 1.2) except the first step when we place it on the non-empty oval (for even $m$ ) or on the odd branch (for odd $m$ ).

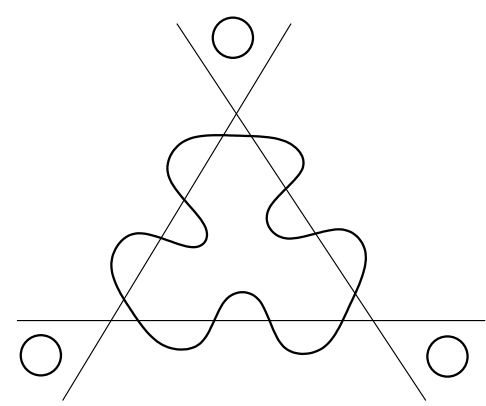

Figure 1.0. The curve $C_{4,0}$

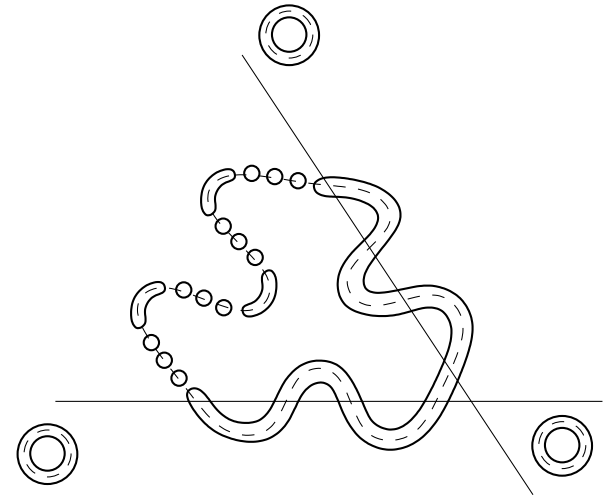

Figure 1.1. The curve $C_{4,1}$

(b). The first three steps of this construction can be performed algebraically if the initial curve is arranged with respect to some three lines as in Figure 1.0. It means that there are three disjoint arcs on the non-empty oval (on the odd branch for odd $m$ ) meeting three lines at $m$ points which lie on the arcs in the same order as on the lines. By classical terminology, such arcs are called bases. 


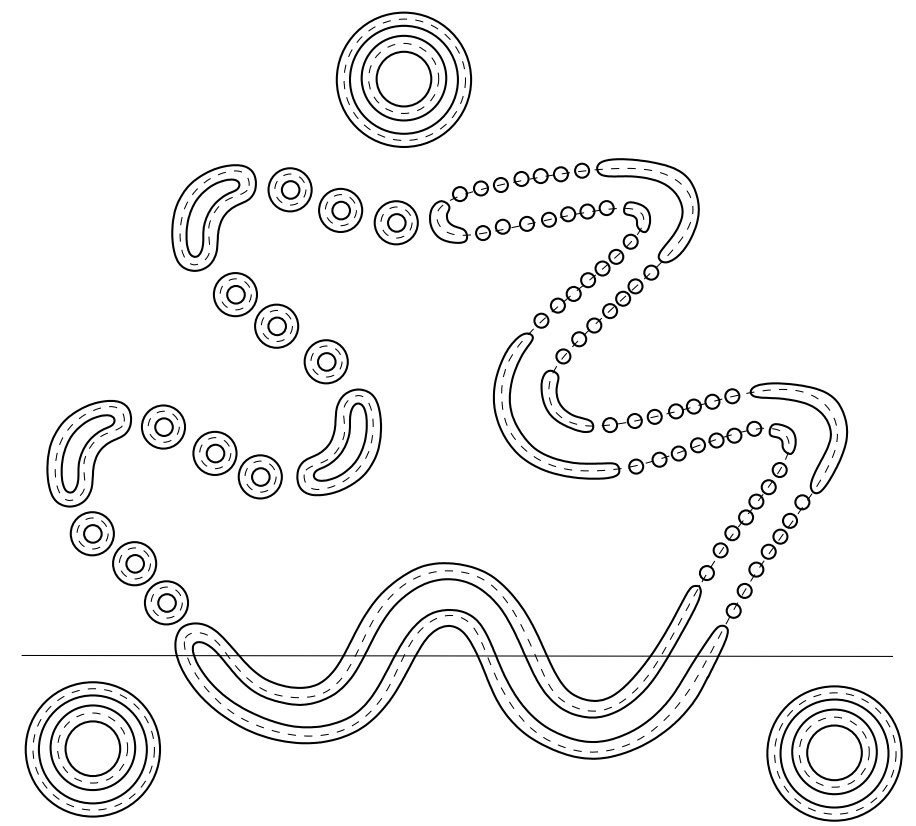

FiguRE 1.2. The curve $C_{4,2}$

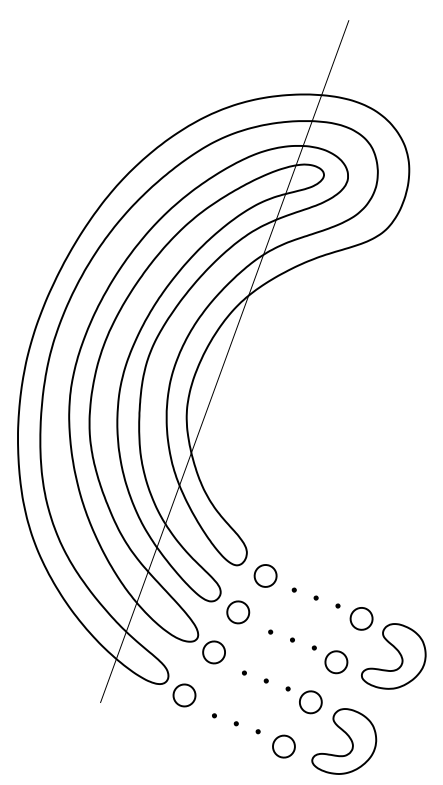

Figure 1.3. A part of $C_{2,3}^{\prime}$

(c). To continue iterations of Wiman's construction, we need more bases. By Mikhalkin's theorem [18], an $M$-curve of degree $d \geq 3$ cannot have more than three bases. So, we start with $d=2$. Choose a conic $C_{2,0}^{\prime}$, disjoint $\operatorname{arcs} \alpha_{1}, \ldots, \alpha_{k}$ on it, and lines $L_{1}, \ldots, L_{k}$ such that $L_{i}$ cuts $\alpha_{i}$ at two points. Let $C_{2, k+1}^{\prime}$ be obtained from $C_{2, k}^{\prime}$ by Wiman's construction using the line $L_{k}$. It happens, however, that it is not enough to have many bases on the initial curve. The construction produces $M$-curves for $k \leq 3$ because the line $L_{k}$ meets only one oval of $C_{2, k-1}^{\prime}, k=1,2,3$. Unfortunately, starting with $k=4$, the line $L_{k}$ meets more than one oval (see Figure 1.3 where we depicted $L_{4}$ and the part of $C_{2,3}^{\prime}$ obtained from that oval of $C_{2,2}^{\prime}$ which meets $\left.L_{3}\right)$. Easy to see that $L_{k}$ meets $2^{k-3}$ ovals for $k \geq 3$. Using this fact, the result can be easily proven by induction.

Lemma 2.2. Let $A$ be a real pseudoholomorphic curve of degree $d=2 k$. Suppose that an empty oval $V$ of $A$ has a tangency of order $d$ with a line L. Let $S$ be the isotopy type of $A \backslash V$. Then there exists a pseudoholomorphic curve $A^{\prime}$ of degree $2 d$ one of whose empty ovals has a tangency of order $2 d$ with $L$, and the isotopy type of $A^{\prime}$ is $S^{2} \sqcup d^{2}$, in particular, if $A$ is an $M$-curve, then $A^{\prime}$ is an $M$-curve also.
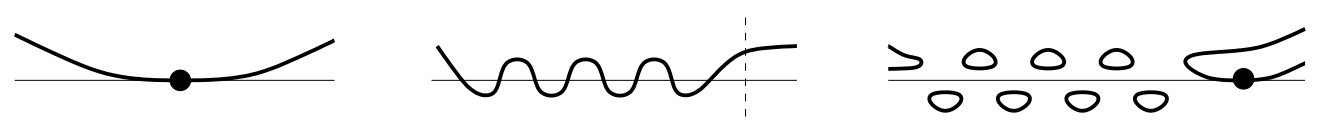

FIGURE 2

Proof. Let $p$ be the tangency point, We apply Wiman's construction in two steps. First, we perturb $A$ so that the perturbed curve $A^{\prime \prime}$ has a tangency with $A$ at $p$ of order $d$ and has $d^{2}-d$ more intersection points, all lying on $V$. We may assume that $A \cup A^{\prime \prime}$ is holomorphic in some neighbourhood of $p$ and is defined by the equation $\left(y-a x^{d}\right)\left(y-b x^{d}\right)=0,0<a<b$. Then we perturb $A \cup A^{\prime \prime}$ by gluing at $p$ the chart $(y-P(x)) y+\varepsilon x^{2 d}$ where roots of $P$ are real negative (see Figure 2). 
Corollary 2.3. For any $d$ there exists a real pseudoholomorphic $M$-curve $A_{d}$ on $\mathbb{R P}^{2}$ of degree $d$ which has at least $L_{d}=\frac{1}{6} d^{2}-\frac{7}{54}(3 d)^{4 / 3}+O(d)$ non-empty ovals.

Proof. Let $k=\left[\frac{1}{3} \log _{2}(3 d)\right]$ and $d=2^{k} m+r, 0 \leq r<2^{k}$. Let $C=C_{m, k}$ be as in Proposition 2.1. By Lemma 2.2, we may suppose that $C$ has a maximal tangency with some line. So, let $A$ be obtained from $C$ by applying $r$ times Harnack's construction. Then $A$ is an $M$-curve and the number of its nonempty ovals is at least $L_{d}=\frac{1}{6}\left(d_{1}^{2}-m^{2}\right)-\frac{3}{2}\left(d_{1}-m\right)+k$ where $d_{1}=2^{k} m=\operatorname{deg} C$. Note that $(x, r)$, $x=2^{k}$, satisfies

$$
(3 d)^{1 / 3} \leq 2 x \leq 2 \cdot(3 d)^{1 / 3}, \quad 0 \leq r \leq x-1
$$

and $L_{d}=\frac{1}{6} f\left(2^{k}, r\right)+k$ where $f(x, r)=(d-r)^{2}\left(1-x^{-2}\right)-9(d-r)\left(1-x^{-1}\right)$. It is an easy calculus exercise to find the minimum of $f$ under the constraints (3).

Remark. It seems that the term $O\left(d^{4 / 3}\right)$ in Corollary 2.3 is not optimal. Maybe, using a more careful construction (like in Section 3) it can be replaced by $O(d)$.

In contrary, it is not clear at all how to construct real algebraic curves of any degree $d$ with $\frac{1}{6} d^{2}+o\left(d^{2}\right)$ non-empty ovals. Proposition 2.1(c) gives an example with this asymptotics for the sequence of degrees $d_{k}=2^{k}$, but is it possible to do the same for, say, $d_{k}=2^{k}-1$ ?

\section{When THE BRAID $\sigma_{1}^{-N} \Delta^{n}$ IS QUASIPOSITIVE}

The purpose of this section is, for given $n$ and $k$, to find $N$ as large as possible such that the braid $\sigma_{1}^{-N} \Delta_{k}^{n}$ is quasipositive (see $\S 3.1$ for definitions and see $\S 4$ and $\S 5$ for motivations). We propose here a recursive construction based on the binary decomposition of $k$. The best value of $N$ obtained by this construction is presented in Theorem 3.13 (see also Corollary 3.15) in $§ 3.6$. We cannot prove that the obtained value of $N$ is optimal.

\subsection{Quasipositive braids.}

Let $B_{n}$ be the group of braids with $n$ strings ( $n$-braids). It is generated by $\sigma_{1}, \ldots, \sigma_{n-1}$, subject to relations $\sigma_{i} \sigma_{j}=\sigma_{j} \sigma_{i}$ for $j-i>1$ and $\sigma_{i} \sigma_{j} \sigma_{i}=\sigma_{j} \sigma_{i} \sigma_{j}$ for $j-i=1$. We suppose that $\{1\}=B_{1} \subset B_{2} \subset B_{3} \subset \ldots$ by identifying $\sigma_{i}$ of $B_{k}$ with $\sigma_{i}$ of $B_{n}$. We set $B_{\infty}=\bigcup_{m} B_{n}$. Let $\Delta_{n}$ be the Garside element of $B_{n}$. It is defined by

$$
\Delta_{0}=\Delta_{1}=1, \quad \Delta_{n+1}=\sigma_{1} \sigma_{2} \ldots \sigma_{n} \Delta_{n} .
$$

Let $Q_{n}$ be the submonoid of $B_{n}$ generated by $\left\{a^{-1} \sigma_{i} a \mid a \in B_{n}, 1 \leq i<n\right\}$. The elements of $Q_{n}$ are called quasipositive braids (this term was introduced by Lee Rudolph in [25]). Theorem 3.1 in $\S 3.3$ shows that $Q_{k+1} \cap B_{k}=Q_{k}$, i.e., the notion of quasipositivity is compatible with the convention that $B_{k} \subset B_{k+1}$.

We introduce a partial order on $B_{n}$ by setting $a \leq b$ if $a b^{-1} \in Q_{n}$. Then $Q_{n}=\left\{x \in B_{n} \mid x \geq 1\right\}$. Since $Q_{n}$ is invariant under conjugation, this order is left and right invariant, i.e. $b^{\prime} \leq b$ implies $a b^{\prime} c \leq a b c$. Indeed, if $b^{\prime} b^{-1} \in Q_{n}$, then $\left(a b^{\prime} c\right)(a b c)^{-1}=a\left(b^{\prime} b^{-1}\right) a^{-1} \in Q_{n}$.

We write $a \sim b$ if $a$ and $b$ are conjugate. Note that $a \sim b \geq c$ does not imply $a \geq c$. Indeed, for $n=3$ we have $\sigma_{2} \sim \sigma_{1} \geq \sigma_{1} \sigma_{2}^{-1}$ but the assertion $\sigma_{2} \geq \sigma_{1} \sigma_{2}^{-1}$ is wrong because $\sigma_{2}\left(\sigma_{1} \sigma_{2}^{-1}\right)^{-1}=\sigma_{2}^{2} \sigma_{1}^{-1} \notin Q P_{3}$ (see, e.g., [20] or [23]). However, $b_{1} \sim b_{2} \geq b_{3} \sim b_{4} \geq \cdots \sim b_{2 n} \geq 1$ does imply $b_{1} \geq 1$. 


\subsection{Shifts and cablings.}

Let $s_{m}, c_{m}: B_{\infty} \rightarrow B_{\infty}$ be the group homomorphisms of $m$-shift and $m$-cabling defined respectively by $s_{m}\left(\sigma_{i}\right)=\sigma_{i+m}$ and

$$
c_{m}\left(\sigma_{i}\right)=\left(\sigma_{m i} \sigma_{m i+1} \ldots \sigma_{m i+m-1}\right)\left(\sigma_{m i-1} \ldots \sigma_{m i+m-2}\right) \ldots\left(\sigma_{m i-m+1} \ldots \sigma_{m i}\right) .
$$

(see the left hand side of Fig. 4). We set $c=c_{2}$ (Fig. 3), $c^{d}=c_{2^{d}}$ and $s^{d}=s_{2^{d}}$. Then

$$
c^{d}=c \circ \cdots \circ c \quad(d \text { times }), \quad c\left(\sigma_{i}\right)=\sigma_{2 i} \sigma_{2 i-1} \sigma_{2 i+1} \sigma_{2 i} .
$$
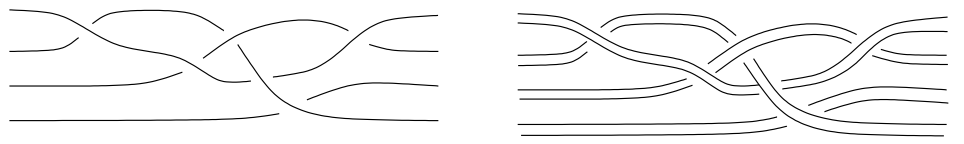

FiguRE 3. Example of 2-cabling: $c\left(\sigma_{3} \sigma_{2} \sigma_{3}^{-1} \sigma_{2} \sigma_{1} \sigma_{3}\right)$
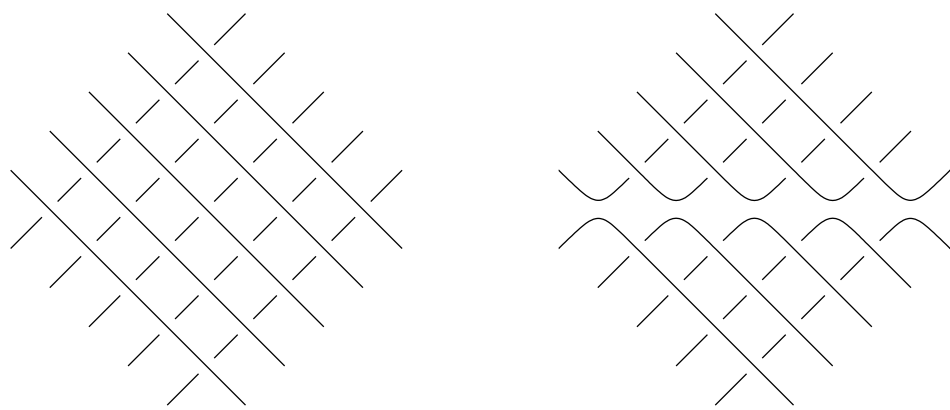

FigURE 4. $c_{k}\left(\sigma_{1}\right) \geq \Delta_{k} \tilde{\Delta}_{k}(k=5)$

Let $r_{m}: B_{m} \rightarrow B_{m}$ be the index reversing homomorphism: $r_{m}\left(\sigma_{j}\right)=\sigma_{m-j}$. Let $\tilde{\Delta}_{n}=s_{n}\left(\Delta_{n}\right)$. Then we have

$$
\begin{gathered}
b \Delta_{m}=\Delta_{m} r_{m}(b), \quad b \in B_{m} ; \quad r_{m}\left(\Delta_{m}\right)=\Delta_{m} \\
\tilde{\Delta}_{k} \Delta_{2 k}=\Delta_{2 k} \Delta_{k}, \quad \Delta_{k} \Delta_{2 k}=\Delta_{2 k} \tilde{\Delta}_{k}, \\
\tilde{\Delta}_{k} c_{k}\left(\sigma_{1}\right)=c_{k}\left(\sigma_{1}\right) \Delta_{k}, \quad \Delta_{k} c_{k}\left(\sigma_{1}\right)=c_{k}\left(\sigma_{1}\right) \tilde{\Delta}_{k}, \\
s_{k i}\left(\Delta_{k}\right) s_{k l}\left(\Delta_{k}\right)=s_{k l}\left(\Delta_{k}\right) s_{k i}\left(\Delta_{k}\right), \\
\Delta_{2 k}=\Delta_{k} \tilde{\Delta}_{k} c_{k}\left(\sigma_{1}\right)=\Delta_{k} c_{k}\left(\sigma_{1}\right) \Delta_{k} .
\end{gathered}
$$

The last identity is the specialization for $a=2$ of

$$
\Delta_{a k}=c_{k}\left(\Delta_{a}\right) \prod_{j=0}^{a-1} s_{j k}\left(\Delta_{k}\right) \text {. }
$$

All these identities easily follow, e.g., from the characterization of $\Delta_{k}$ in [9].

Combining (6)-(9), we obtain

$$
\Delta_{2 k}^{2}=\tilde{\Delta}_{k}^{2} \Delta_{k}^{2} c_{k}\left(\sigma_{1}^{2}\right)
$$

We have $c_{k}\left(\sigma_{1}\right) \geq \Delta_{k} \tilde{\Delta}_{k}$ (see Figure 4). Combining this with (6), we obtain

$$
c_{k}\left(\sigma_{1}\right) \geq \Delta_{k}^{a} \tilde{\Delta}_{k}^{b} \quad \text { for any } a, b \text { such that } a+b=2 \text {. }
$$

Indeed, $c_{k}\left(\sigma_{1}\right) \stackrel{(6)}{=} \Delta_{k}^{a-1} c_{k}\left(\sigma_{1}\right) \tilde{\Delta}_{k}^{1-a} \stackrel{\text { Fig. } 4}{\geq} \Delta_{k}^{a-1}\left(\Delta_{k} \tilde{\Delta}_{k}\right) \tilde{\Delta}_{k}^{1-a}=\Delta_{k}^{a} \tilde{\Delta}_{k}^{2-a}$.

Combining (12) and (9) we obtain also

$$
\Delta_{2 k}=\Delta_{k} c_{k}\left(\sigma_{1}\right) \Delta_{k} \geq \Delta_{k}^{4}
$$




\subsection{Quasipositivity and stabilizations.}

In this section we show that the quasipositivity is stable under two kinds of stabilizations: the inclusion $B_{n} \subset B_{n+1}$ and positive Markov moves.

Theorem 3.1. $Q_{n+1} \cap B_{n}=Q_{n}$.

This is a specialization for $k=1$ of the following fact.

Theorem 3.2. Let $a \in B_{k}, b \in B_{n}$, and $c=s_{n}(a) b \in B_{n+k}$. Suppose that $c \in Q_{n+k}$. Then $a \in Q_{k}$ and $b \in Q_{n}$.

Proof. Let $D$ be the unit disk in $\mathbb{C}$. By Rudolph's theorem [25], a braid is quasipositive if and only if it is cut on $(\partial D) \times \mathbb{C}$ by an algebraic curve in $D \times \mathbb{C}$ which has no vertical asymptote.

Let $L_{a}, L_{b}$, and $L_{c}$ be the links in 3 -sphere represented by $a, b$, and $c$. Let $A_{c}$ be the algebraic curve bounded by $L_{c}$. The fact that $c=s_{n}(a) b$ means that $L_{c}=L_{a} \cup L_{b}$ and the sublinks $L_{a}, L_{b}$ are separated by an embedded sphere. Then, by Eroshkin's theorem [10], $A_{c}$ is a disjoint union of curves $A_{a}$ and $A_{b}$ bounded by $L_{a}$ and $L_{b}$ respectively. Hence, $a$ and $b$ are quasipositive.

This proof of Theorem 3.2 relies on analytic methods (the filling disk techniques is the main tool in [10]). However, Theorem 3.1 has a purely combinatorial proof based on Dehornoy's results [8] completed by Burckel-Laver's theorem [3,17].

Say that a braid $b \in B_{n}$ is Dehornoy $i$-positive, ${ }^{1} i=1, \ldots, n-1$, if there exist braids $b_{0}, \ldots, b_{k} \in B_{n-i}, k \geq 1$, such that $b=b_{0} \prod_{j=0}^{k}\left(\sigma_{n-i} b_{j}\right)$. Say that $b$ is Dehornoy positive if it is $i$-positive for some $i=1, \ldots, n-1$. Let $P_{i}$ be the set of $(n+1-i)$-positive braids and $\bar{P}_{i}=\bigcup_{j=1}^{i} P_{j}$.

In this notation, Dehornoy's theorem [8] (see also [11] for another proof) states that $(i) B_{n}$ is a disjoint union $\{1\} \cup \bar{P}_{n} \cup \bar{P}_{n}^{-1} ;(i i) \bar{P}_{n}$ is a disjoint union $P_{2} \cup \cdots \cup P_{n}$; (iii) $P_{i}$ and $\bar{P}_{i}, 2 \leq i \leq n$, are subsemigroups of $B_{n}$. Burckel-Laver's theorem [3, 17] (see also [20] or [34] for another proof) states that (iv) $Q_{n} \subset \bar{P}_{n}$.

Combinatorial proof of Theorem 3.1. The inclusion $Q_{n} \subset Q_{n+1} \cap B_{n}$ is evident. Let us show that $Q_{n+1} \cap B_{n} \subset Q_{n}$. Let $b \in Q_{n+1} \cap B_{n}$. Then $b=x_{1} \ldots x_{k}$, each $x_{j}$ being a conjugate of $\sigma_{1}$ in $B_{n+1}$. By $(i v)$, we have $x_{j} \in \bar{P}_{n+1}, j=1, \ldots, k$. If $x_{j} \in P_{n+1}$ for some $j$, then $b \in P_{n+1}$ by definition of $i$-positivity. By $(i i)$, this contradicts $b \in B_{n}$. Hence, each $x_{j}$ is in $P_{n}$.

Thus, it remains to show that if $x$ is a conjugate of $\sigma_{1}$ in $B_{n+1}$, then $x$ is a conjugate of $\sigma_{1}$ in $B_{n}$. This follows from the fact that any conjugate of $\sigma_{1}$ can be presented in a unique way as $x=c a_{i, j} c^{-1}, i<j$, where $a_{i, j}$ is so-called bandgenerator (i.e., $a_{i, j}=a \sigma_{i} a^{-1}$ for $a=\sigma_{j-1} \sigma_{j-2} \ldots \sigma_{i+1}$ ) and $c$ is in the kernel of the pure braid group homomorphism of forgetting the $i$-th string. The latter fact can be easily proved using the braid combing theory.

Stability under positive Markov moves.

Theorem 3.3. Let $b \in B_{n}$. Then $b \in Q_{n}$ if and only if $b \sigma_{n} \in Q_{n+1}$.

This fact is reduced in [21] to Gromov's theorem on pseudoholomorphic curves. The reduction given in [21] is rather cumbersome, but Michel Boileau observed that it can be considerably simplified using the arguments from our joint paper [2]

\footnotetext{
${ }^{1}$ Our definitions differ from those in [8] only by the reversing of the string numbering.
} 
(unfortunately, this observation was done when [2] had already been published). Indeed, it is proved (though not stated explicitly) in [2] that if $L$ is the boundary link of an analytic curve in $B^{4} \subset \mathbb{C}^{2}$, and $L$ is transversally isotopic ${ }^{2}$ to a closed braid $b$, then $b$ is quasipositive. To deduce Theorem 3.3 from this fact, we note that $b \sigma_{n}$ bounds an analytic curve (by Rudolph's theorem [25]), and $b$ is transversally isotopic to $b \sigma_{n}$ (an easy exercise; see, e.g., [24; Lemma 1]).
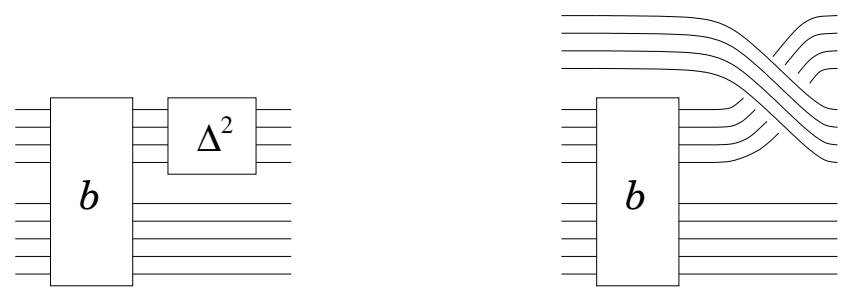

Figure 5. The braids $b^{\prime}$ (on the left) and $b^{\prime \prime}$ (on the right)

Corollary 3.4. Let $b \in B_{n}$ and $k \leq n$. Then $b^{\prime}=b s_{n-k}\left(\Delta_{k}^{2}\right)$ is quasipositive if and only if $b^{\prime \prime}=b s_{n-k}\left(c_{k}\left(\sigma_{1}\right)\right)$ is quasipositive (see Figure 5).
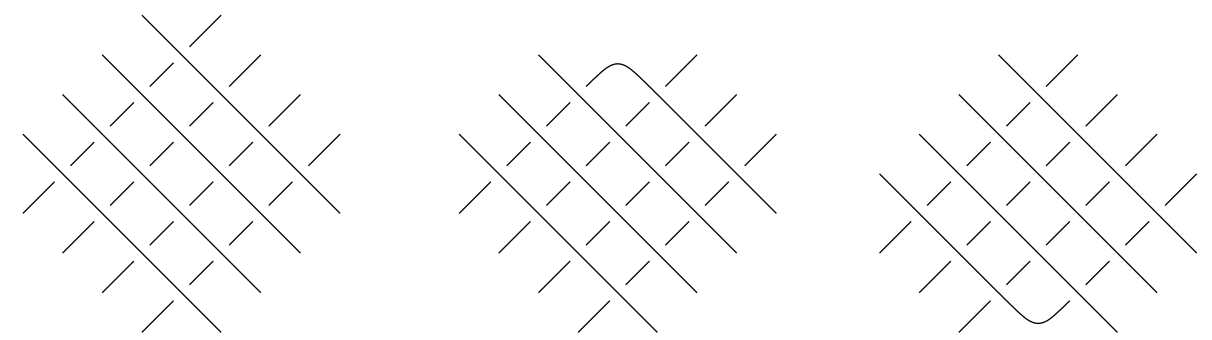

FiguRE 6. $c_{k+1}\left(\sigma_{1}\right) \stackrel{M m}{\rightarrow} \cdots=\left(\sigma_{k} \ldots \sigma_{2} \sigma_{1}\right) \cdot s_{1}\left(c_{k}\left(\sigma_{1}\right)\right) \cdot\left(\sigma_{1} \sigma_{2} \ldots \sigma_{k}\right)$

Proof. We say that $b_{1} b_{2}$ is obtained from $b_{0}$ by a positive Markov move (and we write $\left.b_{0} \stackrel{M m}{\rightarrow} b_{1} b_{2}\right)$ if $b_{1}, b_{2} \in B_{n}$ and $b_{0}=b_{1} \sigma_{n} b_{2}$. By Theorem 3.3, it is enough to prove that $b^{\prime \prime} \stackrel{M m}{\rightarrow} \ldots \stackrel{M m}{\rightarrow} b^{\prime}$. If $k=0$, this is trivial. Suppose that this statement is proved for $k$. Then

$$
\begin{array}{rlr}
c_{k+1}\left(\sigma_{1}\right) & \stackrel{M m}{\rightarrow}\left(\sigma_{k} \ldots \sigma_{1}\right) s_{1}\left(c_{k}\left(\sigma_{1}\right)\right)\left(\sigma_{1} \ldots \sigma_{k}\right) & \text { (see Figure 6) } \\
& \stackrel{M m}{\rightarrow}\left(\sigma_{k} \ldots \sigma_{1}\right) s_{1}\left(\Delta_{k}^{2}\right)\left(\sigma_{1} \ldots \sigma_{k}\right) & \text { (by the induction hypothesis) } \\
& =r_{k+1}\left(\sigma_{1} \ldots \sigma_{k} \Delta_{k}^{2} \sigma_{k} \ldots \sigma_{1}\right) \stackrel{(4)}{=} \Delta_{k+1}^{2} . \quad \square
\end{array}
$$

\subsection{Subgroup $A_{\infty}$ of $B_{\infty}$.}

For and integer $d \geq 1$, let $X_{d}=\left\{s_{k 2^{d}}\left(\Delta_{2^{d}}\right) \mid k \geq 0, k \in \mathbb{Z}\right\}$ and let $A_{d}$ be the subgroup of $B_{\infty}$ generated by $X_{d}$. It is a free abelian group freely generated by $X_{d}$. For example, $A_{1}$ is the subgroup of $B_{\infty}$ generated by $\sigma_{1}, \sigma_{3}, \sigma_{5}, \ldots$

Let $A_{\infty}$ be the subgroup of $B_{\infty}$ generated by $\bigcup X_{d}$, i.e., the product of all the subgroups $A_{d}$. This product is semidirect in the sense that $A_{1} \ldots A_{d}$ is a normal

\footnotetext{
${ }^{2}$ In the sense of the contact geometry
} 
subgroup of $A_{\infty}$, and for any $d, e$, the subgroup $A_{e}$ is a normal in $A_{e} A_{d}$ if $e \leq d$. In the latter case, the action of $A_{d}$ on $A_{e}$ by conjugation is very easy to describe. Let $x \in X_{e}, y \in X_{d}, e \leq d$. Let $P_{x}$ (resp. $P_{y}$ ) be the set of strings permuted by $x$ (resp. by $y$ ). Only two cases are possible: either $P_{x}$ and $P_{y}$ are disjoint and then $x$ and $y$ commute, or $P_{x} \subset P_{y}$ and then $y$ acts on $x$ as in (5).

In particular, each element $x$ of $A_{1} \ldots A_{d}$ can be uniquely presented in the form

$$
x=x_{1} \ldots x_{d}, \quad x_{e} \in A_{e}
$$

Let $\chi_{d}: A_{d} \rightarrow \mathbb{Z}$ be the homomorphism which takes each element of $X_{d}$ to 1 , and let $A_{d}^{m}=\chi_{d}^{-1}(m)$. Since $A_{\infty}$ is a semidirect product of $A_{d}$ 's, the characters $\chi_{d}$ extend in a unique way to a homomorphism $\chi: A_{\infty} \rightarrow \bigoplus_{d=1}^{\infty} \mathbb{Z}$ such that $\chi\left(x_{1} \ldots x_{d}\right)=\left(\chi_{1}\left(x_{1}\right), \ldots, \chi_{d}\left(x_{d}\right)\right)$ if $x_{e} \in A_{e}$ for $e=1, \ldots, d$ (here and below, we truncate the tail of zeros).

The above discussion implies also the following two easy facts:

Lemma 3.5. Let $0<r<2^{d}$ and $m=2^{d} q+r$. Then $A_{\infty} \cap B_{m}$ is the direct product of its subgroups $A_{\infty} \cap B_{m-r}$ and $s_{m-r}\left(A_{\infty} \cap B_{r}\right)$.

Lemma 3.6. Let $B=B_{2^{d}}, \tilde{B}=s^{d}(B)$. Let $x \in A_{\infty} \cap B_{2^{d+1}}$ and $n=\left(n_{1}, \ldots, n_{d}\right)=$ $\chi(x)$. Then, for any decomposition $n=n^{\prime}+n^{\prime \prime}+\tilde{n}^{\prime}+\tilde{n}^{\prime \prime}$, there exist $x^{\prime}, x^{\prime \prime} \in B$ and $\tilde{x}^{\prime}, \tilde{x}^{\prime \prime} \in \tilde{B}$ such that $\chi\left(x^{\prime}\right)=n^{\prime}, \chi\left(x^{\prime \prime}\right)=n^{\prime \prime}, \chi\left(\tilde{x}^{\prime}\right)=\tilde{n}^{\prime}, \chi\left(\tilde{x}^{\prime \prime}\right)=\tilde{n}^{\prime \prime}$, and

$$
x \Delta_{2^{d+1}}^{2 n+1} \sim x^{\prime} \tilde{x}^{\prime} \Delta_{2^{d+1}}^{2 n+1} x^{\prime \prime} \tilde{x}^{\prime \prime} .
$$

Proof. (hope that the notation is self-explained)

$$
x \Delta_{2^{d+1}}^{2 n+1}=a b c \tilde{u} \tilde{v} \tilde{w} \Delta_{2^{d+1}}^{2 n+1}=a \tilde{u} \Delta_{2^{d+1}}^{2 n+1} v w \tilde{b} \tilde{c} \sim w a \tilde{c} \tilde{u} \Delta_{2^{d+1}}^{2 n+1} v \tilde{b}
$$

\subsection{The case when the number of strings is a power of 2 .}

For any $d \geq 0$, we set

$$
S_{d}=1+4+4^{2}+\cdots+4^{d-1}=\left(4^{d}-1\right) / 3 .
$$

So, $\left(S_{0}, S_{1}, \ldots\right)=(0,1,5,21,85,341,1365, \ldots)$. We have the recurrences:

$$
S_{d}-4 S_{d-1}=1, \quad S_{d}-5 S_{d-1}+4 S_{d-2}=0 .
$$

Lemma 3.7. Let $x \in A_{\infty} \cap B_{2^{d}}, \chi(x)=\left(n_{1}, \ldots, n_{d}\right)$. If $d=1$, we suppose only that $n_{1} \geq 0$. If $d \geq 2$, we suppose that

$$
\sum_{e=k+1}^{d}\left(n_{e} S_{e-k}-\varepsilon_{e}\right) \geq 0, \quad k=0, \ldots, d-1,
$$

where

$$
\varepsilon_{1}=1, \quad \varepsilon_{d}=\frac{3+(-1)^{n_{d}}}{2}, \quad \varepsilon_{e}=\frac{5-(-1)^{n_{e}}}{2}, \quad 1<e<d,
$$

i.e., $n_{d} \geq \varepsilon_{d}, 5 n_{d}+n_{d-1} \geq \varepsilon_{d}+\varepsilon_{d-1}, \ldots, S_{d} n_{d}+\cdots+5 n_{2}+n_{1} \geq \varepsilon_{d}+\cdots+\varepsilon_{1}$.

Then $x$ is quasipositive. 
Proof. Induction by $d$. If $d=1$, then the statement is trivial because in this case $x=\sigma_{1}^{n_{1}}$. So, let us assume that the statement is true for $d-1$ and let us prove it for $d$.

Let $\Delta=\Delta_{2^{d-1}}, \tilde{\Delta}=\tilde{\Delta}_{2^{d-1}}=s^{d-1}(\Delta), \delta_{k}=s_{(k-1) 2^{d-2}}\left(\Delta_{2^{d-2}}\right), \hat{\sigma}_{k}=c^{d-2}\left(\sigma_{k}\right)$. The notation $\delta_{12}^{a}$ is an abbreviation for $\delta_{1}^{a^{\prime}} \delta_{2}^{a-a^{\prime}}$ when the value of $a^{\prime}$ is not important for us. In this notation, (6) - (9) and (12) specialize to

$$
\begin{gathered}
\Delta \Delta_{2^{d}}=\Delta_{2^{d}} \tilde{\Delta}, \quad \delta_{1} \Delta=\Delta \delta_{2}, \quad \delta_{3} \tilde{\Delta}=\tilde{\Delta} \delta_{4}, \\
\hat{\sigma}_{i} \delta_{i}=\delta_{i+1} \hat{\sigma}_{i}, \quad \hat{\sigma}_{i} \delta_{i+1}=\delta_{i} \hat{\sigma}_{i}, \quad \hat{\sigma}_{i} \delta_{k}=\delta_{k} \hat{\sigma}_{i}, \quad k \notin\{i, i+1\} . \\
\delta_{i} \delta_{l}=\delta_{l} \delta_{i}, \quad \Delta \tilde{\Delta}=\tilde{\Delta} \Delta, \\
\Delta=\hat{\sigma}_{1} \delta_{1} \delta_{2} \quad \tilde{\Delta}=\hat{\sigma}_{3} \delta_{3} \delta_{4}, \\
\forall a \in \mathbb{Z}, \quad \hat{\sigma}_{k} \geq \delta_{k}^{a} \delta_{k+1}^{2-a}
\end{gathered}
$$

Combining $\left(12^{\prime}\right)$ and (9) we obtain

$$
\hat{\sigma}_{1} \hat{\sigma}_{2} \stackrel{(12)}{\geq} \hat{\sigma}_{1} \delta_{2}^{2} \stackrel{(9)}{=} \Delta \delta_{1}^{-1} \delta_{2}=\Delta \delta_{12}^{0}
$$

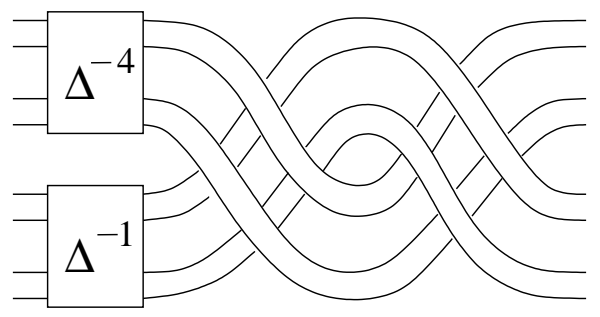

FiguRE 7.1. $\tilde{\Delta}^{-6} \Delta^{-3} \Delta_{2^{d}}^{2}=\ldots$

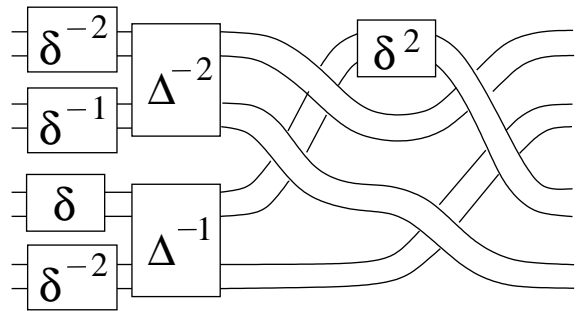

FiguRE 7.3.

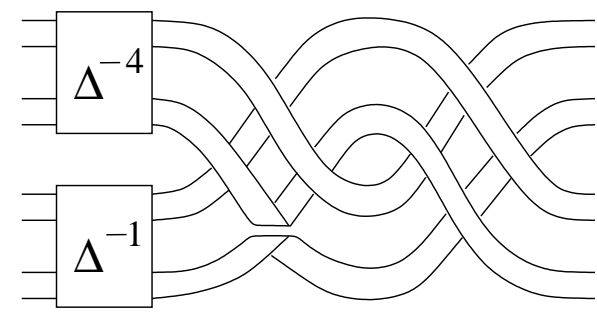

FIGURE 7.2

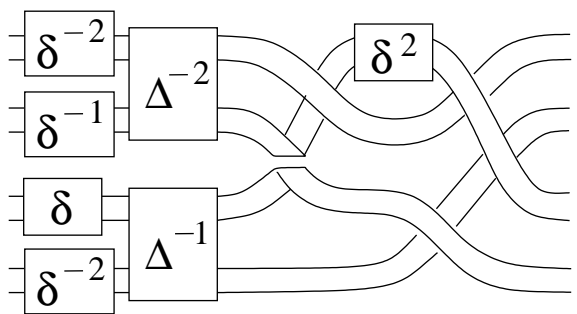

Figure 7.4. $\cdots=\delta_{1}^{-2} \delta_{4}^{-4} \hat{\sigma}_{2}$

Let us show that

$$
\tilde{\Delta}^{-6} \Delta^{-3} \Delta_{2^{d}}^{2} \geq \delta_{1}^{-2} \delta_{4}^{-4} \hat{\sigma}_{2}
$$

(this is the heart of the proof). Indeed (see Figures $7.1-7.4$ ),

$$
\begin{aligned}
\tilde{\Delta}^{-6} \Delta^{-3} & \Delta_{2^{d}}^{2} \stackrel{(11)}{=} \tilde{\Delta}^{-6} \Delta^{-3}\left(\Delta^{2} \tilde{\Delta}^{2} c^{d-1}\left(\sigma_{1}^{2}\right)\right)=\tilde{\Delta}^{-4} \Delta^{-1}\left(\hat{\sigma}_{2} \hat{\sigma}_{1} \hat{\sigma}_{3} \hat{\sigma}_{2}\right)^{2} \\
& \stackrel{(12)}{\geq} \tilde{\Delta}^{-4} \Delta^{-1} \hat{\sigma}_{2}\left(\delta_{1}^{2-a} \delta_{2}^{a}\right) \hat{\sigma}_{3} \hat{\sigma}_{2}^{2} \hat{\sigma}_{3} \hat{\sigma}_{1} \hat{\sigma}_{2} \stackrel{(7)}{=} \tilde{\Delta}^{-4} \Delta^{-1}\left(\hat{\sigma}_{2} \hat{\sigma}_{3} \hat{\sigma}_{2}^{2}\right) \hat{\sigma}_{3} \hat{\sigma}_{1} \delta_{1}^{a} \delta_{2}^{2-a} \hat{\sigma}_{2} \\
& =\tilde{\Delta}^{-4} \Delta^{-1} \hat{\sigma}_{3}^{2} \sigma_{2} \hat{\sigma}_{3}^{2} \hat{\sigma}_{1} \delta_{1}^{a} \delta_{2}^{2-a} \hat{\sigma}_{2} \stackrel{(12)}{\geq} \tilde{\Delta}^{-4} \Delta^{-1} \hat{\sigma}_{3}^{2}\left(\delta_{2}^{b} \delta_{3}^{2-b}\right) \hat{\sigma}_{3}^{2} \hat{\sigma}_{1} \delta_{1}^{a} \delta_{2}^{2-a} \hat{\sigma}_{2} \\
& \stackrel{(7)}{=} \tilde{\Delta}^{-4} \Delta^{-1} \hat{\sigma}_{3}^{4} \hat{\sigma}_{1} \delta_{1}^{a+b} \delta_{2}^{2-a} \delta_{3}^{2-b} \hat{\sigma}_{2} \stackrel{(9)}{=}\left(\delta_{1} \delta_{2}\right)^{-1}\left(\delta_{3} \delta_{4}\right)^{-4} \delta_{1}^{a+b} \delta_{2}^{2-a} \delta_{3}^{2-b} \hat{\sigma}_{2}
\end{aligned}
$$


and we obtain (19) by setting $a=1, b=-2$. We have also

$$
\Delta_{2^{d}} \geq \hat{\sigma}_{1} \hat{\sigma}_{2} \Delta^{2} \delta_{12}^{4}
$$

Indeed,

$$
\begin{aligned}
\Delta_{2^{d}} & \stackrel{(9)}{=} \Delta c^{d-1}\left(\sigma_{1}\right) \Delta=\Delta \hat{\sigma}_{2} \hat{\sigma}_{1} \hat{\sigma}_{3} \hat{\sigma}_{2} \Delta \stackrel{(12)}{\geq} \Delta \hat{\sigma}_{2} \hat{\sigma}_{1}\left(\delta_{3}^{2}\right)\left(\delta_{2}^{5} \delta_{3}^{-3}\right) \Delta \\
& \stackrel{(9)}{=} \hat{\sigma}_{1} \delta_{1} \delta_{2} \hat{\sigma}_{2} \hat{\sigma}_{1} \delta_{2}^{5} \delta_{3}^{-1} \Delta \stackrel{(7)}{=} \hat{\sigma}_{1} \hat{\sigma}_{2} \hat{\sigma}_{1} \delta_{2}^{6} \Delta \stackrel{(9)}{=} \hat{\sigma}_{1} \hat{\sigma}_{2}\left(\Delta \delta_{1}^{-1} \delta_{2}^{-1}\right) \delta_{2}^{6} \Delta \stackrel{(9)}{=} \hat{\sigma}_{1} \hat{\sigma}_{2} \Delta^{2} \delta_{12}^{4} .
\end{aligned}
$$

We set $n_{d}=2 n+1+r, r \in\{0,1\}$. Let $m_{d-1}=n_{d-1}+10 n+4 r, m_{d-2}=n_{d-2}-8 n$,

$$
\begin{aligned}
& n_{d-1}^{\prime}=m_{d-1}+3=n_{d-1}+5 n_{d}-r-2=n_{d-1}+5 n_{d}-\varepsilon_{d}-1, \\
& n_{d-2}^{\prime}=m_{d-2}+4=n_{d-2}-4 n_{d}+4 r+8=n_{d-2}-4 n_{d}+4 \varepsilon_{d}+4,
\end{aligned}
$$

and $n_{e}^{\prime}=n_{e}$ for $e=1, \ldots, d-3$. In the following computation we assume that $y_{1}, y_{2}, z, x^{\prime} \in A_{\infty} \cap B_{2^{d-1}}$ and $\chi\left(y_{1}\right)=\chi\left(y_{2}\right)=\left(n_{1}, \ldots, n_{d-2}, m_{d-1}\right), \chi(z)=$ $\left(n_{1}, \ldots, n_{d-3}, m_{d-2}, m_{d-1}\right), \chi\left(x^{\prime}\right)=\left(n_{1}^{\prime}, \ldots, n_{d-1}^{\prime}\right)$. Let $x=x_{1} \Delta_{2^{d}}^{n_{d}}$ with $x_{1} \in$ $\left(A_{1} \ldots A_{d-1}\right) \cap B_{2^{d}}$. So, we have

$$
\begin{aligned}
x & =x_{1} \ldots x_{d-1} \Delta_{2^{d}}^{n_{d}} \stackrel{(13)}{\geq} x_{1} \ldots x_{d-1} \Delta^{4 r} \Delta_{2^{d}}^{2 n+1} \stackrel{(14)}{\sim} y_{1} \Delta^{-3 n} \tilde{\Delta}^{-6 n} \Delta_{2^{d}}^{2 n} \Delta_{2^{d}} \Delta^{-n} \\
& =y_{1}\left(\Delta^{-3} \tilde{\Delta}^{-6} \Delta_{2^{d}}^{2}\right)^{n} \Delta_{2^{d}} \Delta^{-n} \stackrel{(19)}{\geq} y_{1} \delta_{1}^{-2 n} \delta_{4}^{-4 n} \hat{\sigma}_{2}^{n} \Delta_{2^{d}} \Delta^{-n} \\
& =y_{1} \delta_{1}^{-2 n} \hat{\sigma}_{2}^{n} \Delta_{2^{d}} \delta_{1}^{-4 n} \Delta^{-n} \sim y_{2} \delta_{12}^{-6 n} \hat{\sigma}_{2}^{n} \Delta_{2^{d}} \Delta^{-n} \stackrel{(9)}{=} y_{2} \delta_{12}^{-6 n} \hat{\sigma}_{2}^{n} \Delta_{2^{d}} \hat{\sigma}_{1}^{-n} \delta_{12}^{-2 n} \\
& \sim z \hat{\sigma}_{2}^{n} \Delta_{2^{d}} \hat{\sigma}_{1}^{-n} \stackrel{(20)}{\geq} z \hat{\sigma}_{2}^{n} \hat{\sigma}_{1} \hat{\sigma}_{2} \delta_{12}^{4} \Delta^{2} \hat{\sigma}_{1}^{-n}=z \hat{\sigma}_{1} \hat{\sigma}_{2} \delta_{12}^{4} \Delta^{2} \stackrel{(18)}{\geq} z \delta_{12}^{4} \Delta^{3}=x^{\prime} .
\end{aligned}
$$

It remains to check that the induction conditions are satisfied for $x^{\prime}$ and $d-1$. If $d=2$, then $n_{1}^{\prime}=n_{1}+5 n_{2}-\varepsilon_{2}-1=\left(n_{1} S_{1}-\varepsilon_{1}\right)+\left(n_{2} S_{2}-\varepsilon_{2}\right) \geq 0$ and we are done.

Suppose that $d>2$. Let $\left(16^{\prime}\right)$ and $\left(17^{\prime}\right)$ refer to the formulas (16), (17) where $d-1, n_{e}^{\prime}$, and $\varepsilon_{e}^{\prime}$ replace $d, n_{e}$, and $\varepsilon_{e}$. So, we define $\varepsilon_{1}^{\prime}, \ldots, \varepsilon_{d-1}^{\prime}$ by $\left(17^{\prime}\right)$ and we have to check the inequalities $\left(16^{\prime}\right)$ for $k=0, \ldots, d-2$. Indeed, we have $n_{e}^{\prime}=n_{e}$ for $e<d-2 ; n_{d-2}^{\prime}-n_{d-2}=-8 n+4$ is even, and $n_{d-1}^{\prime}-n_{d-1}=10 n+4 r+3$ is odd. Hence, $\varepsilon_{e}^{\prime}=\varepsilon_{e}$ for $e \leq 2$, and

$$
\varepsilon_{d-1}^{\prime}=\left(3+(-1)^{n_{d-1}^{\prime}}\right) / 2=\left(3-(-1)^{n_{d-1}}\right) / 2=\left(5-(-1)^{n_{d-1}}\right) / 2-1=\varepsilon_{d-1}-1
$$

and we obtain for any $k=0, \ldots, d-2$

$$
\sum_{e=k+1}^{d} \varepsilon_{e}-\sum_{e=k+1}^{d-1} \varepsilon_{e}^{\prime}=\varepsilon_{d-1}+\varepsilon_{d}-\varepsilon_{d-1}^{\prime}=\varepsilon_{d}+1 .
$$

Since $n_{e}^{\prime}=n_{e}$ for $e<d-2$, and $S_{0}=0$, we have for any $k=d-p \leq d-2$

$$
\begin{aligned}
& \sum_{e=k+1}^{d} n_{e} S_{e-k}-\sum_{e=k+1}^{d-1} n_{e}^{\prime} S_{e-k}=\left(n_{d-2}-n_{d-2}^{\prime}\right) S_{p-2}+\left(n_{d-1}-n_{d-1}^{\prime}\right) S_{p-1}+n_{d} S_{p} \\
& =\left(4 n_{d}-4 \varepsilon_{d}-4\right) S_{p-2}+\left(-5 n_{d}+\varepsilon_{d}+1\right) S_{p-1}+n_{d} S_{p} \\
& =\left(S_{p}-5 S_{p-1}+4 S_{p-2}\right) n_{d}+\left(S_{p-1}-4 S_{p-2}\right)\left(\varepsilon_{d}+1\right) \stackrel{(15)}{=} \varepsilon_{d}+1 .
\end{aligned}
$$

Thus, $\left(16^{\prime}\right)$ is equivalent to $(16)$.

Let us emphasize some particular cases of Lemma 3.7: 
Corollary 3.8. Let $x \in A_{\infty} \cap B_{2^{d}}, d \geq 2, \chi(x)=\left(n_{1}, \ldots, n_{d}\right)$, and let $\varepsilon_{1}, \ldots, \varepsilon_{d}$ be as in (17).

(a). If $n_{d}>0, n_{e} \geq 0$ for $e=2, \ldots, d-1$, and (16) holds for $k=0$, i.e., $\sum_{e}\left(n_{e} S_{e}-\varepsilon_{e}\right) \geq 0$, then $x$ is quasipositive,

(b). In particular, if $n_{2}, \ldots, n_{d}$ are even and non-negative, $n_{d}$ is positive, and

$$
n_{1}+5 n_{2}+21 n_{3}+\cdots+S_{d} n_{d} \geq 2 d-1
$$

then $x$ is quasipositive.

Proof. (a). It is enough to check (16) for $k=1, \ldots, d-1$. First, note that (16) for $k=d-1$ is just $n_{d} \geq \varepsilon_{d}$ which is equivalent to $n_{d}>0$. So, let $1 \leq k \leq d-2$. For any $m \geq 1$ we have $3(m-1) \leq S_{m}-1$. Hence, $\varepsilon_{k+1}+\cdots+\varepsilon_{d-1} \leq 3+\cdots+3=$ $3(d-k-1) \leq S_{d-k}-1 \leq n_{d}\left(S_{d-k}-1\right)$. Thus,

$$
\sum_{e=k+1}^{d}\left(n_{e} S_{e-k}-\varepsilon_{e}\right)=\left(n_{d}\left(S_{d-k}-1\right)-\sum_{e=k+1}^{d-1} \varepsilon_{e}\right)+\left(n_{d}-\varepsilon_{d}\right)+\sum_{e=k+1}^{d-1} S_{e-k} n_{e} \geq 0 .
$$

(b). Immediate from (a).

Corollary 3.9. For positive integers $d, n$, if $N \leq\left(4^{d}-1\right) n / 3-2 d+\left(3-(-1)^{n}\right) / 2$, then $\sigma_{1}^{-N} \Delta_{2^{d}}^{n} \geq 0$.

Proof. $\chi\left(\sigma_{1}^{-N} \Delta_{2^{d}}^{n}\right)=(-N, 0, \ldots, 0, n)$, so we may apply Corollary 3.8.

Remark. Corollary 3.8 combined with arguments similar to those in the proof of Corollary 2.3 allows to show that for any $k$, the braid $\sigma_{1}^{-N} \Delta_{k}$ is quasipositive for $N=1 / 3 k^{2}+O\left(k^{4 / 3}\right)$. However, in the next subsection we give a better estimate for $N$ of the form $1 / 3 k^{2}+O(k)$.

\subsection{The general case.}

Lemma 3.10. Let $p, d>0, m^{\prime}=2^{d} p, m=m^{\prime}+2^{d-1}=(2 p+1) 2^{d-1}$, and $x \in A_{\infty} \cap B_{m}$. Then $x \Delta_{m} \geq x^{\prime} \Delta_{m^{\prime}}$ for some $x^{\prime} \in A_{\infty} \cap B_{m^{\prime}}$ such that $\chi_{d-1}\left(x^{\prime}\right)=$ $\chi_{d-1}(x)+1, \chi_{d}\left(x^{\prime}\right)=\chi_{d}(x)+p$, and $\chi_{e}\left(x^{\prime}\right)=\chi_{e}(x)$ for $e \notin\{d-1, d\}$.

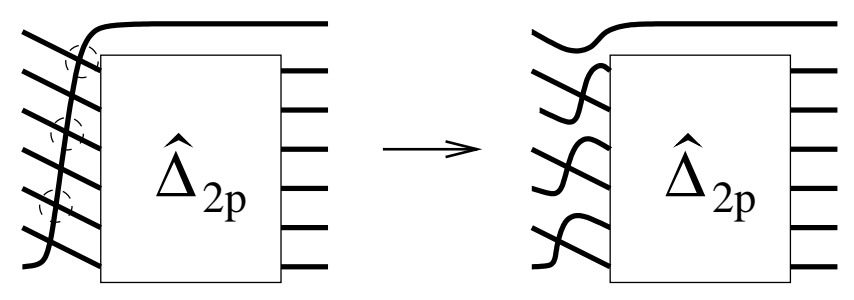

FIGURE 8. Illustration to the proof of Lemma $3.10(p=3)$

Proof. By Lemma 3.5, we may write $x=y \tilde{y}$ with $y \in A_{\infty} \cap B_{m^{\prime}}$, and $\tilde{y} \in A_{\infty} \cap$ $s_{m^{\prime}}\left(B_{2^{d-1}}\right)$. Let $\delta_{k}=s_{2^{d-1}(k-1)}\left(\Delta_{2^{d-1}}\right), \Delta=\Delta_{2^{k}}$, We denote here $c^{d-1}(\alpha)$ by $\hat{\alpha}$ for any braid $\alpha$.

Let $z=\Delta_{m} \tilde{y} \Delta_{m}^{-1}$ and $w=\Delta_{m^{\prime}} z \Delta_{m^{\prime}}^{-1}$. Then, by (5), we have $z, w \in A_{\infty} \cap B_{m^{\prime}}$ and $\chi(w)=\chi(z)=\chi(y)$. In the following computation, the "wild character" $\delta^{a}$ stands for any product of the form $\delta_{1}^{a_{1}} \ldots \delta_{2 p}^{a_{2 p}}\left(\right.$ no $\delta_{2 p+1}$ !) with $a_{1}+\cdots+a_{2 p}=a$ 
when the explicit values of $a_{j}$ 's are not important for us. In other words, $\delta^{a}$ stands for any element of $X_{2^{d-1}}^{a} \cap B_{m^{\prime}}$. Similarly, $\Delta^{a}$ stands for any element of $X_{2^{d}}^{a} \cap B_{m^{\prime}}$. So, we have (see Figure 8)

$$
\begin{aligned}
x \Delta_{m} & =y \tilde{y} \Delta_{m}=y \Delta_{m} z \stackrel{(10)}{=} y \hat{\Delta}_{2 p+1} \delta^{2 p} \delta_{2 p+1} z \stackrel{(5)}{=} y \delta_{1} \hat{\Delta}_{2 p+1} \delta^{2 p} z \\
& \stackrel{(4)}{=} y \delta_{1} \hat{\sigma}_{1} \ldots \hat{\sigma}_{2 p} \hat{\Delta}_{2 p} \delta^{2 p} z \stackrel{(10)}{=} y \delta_{1}\left(\hat{\sigma}_{1} \ldots \hat{\sigma}_{2 p}\right) \Delta_{m^{\prime}} \delta^{0} z \\
& \stackrel{(12)}{\geq} y \delta^{1}\left(\hat{\sigma}_{1} \delta_{2}^{2} \hat{\sigma}_{3} \delta_{4}^{2} \ldots \hat{\sigma}_{2 p-1} \delta_{2 p}^{2}\right) \Delta_{m^{\prime}} z=y \delta^{2 p+1} \hat{\sigma}_{1} \hat{\sigma}_{3} \ldots \hat{\sigma}_{2 p-1} w \Delta_{m^{\prime}} \\
& \stackrel{(9)}{=} y \delta^{1} \Delta^{p} w \Delta_{m^{\prime}} .
\end{aligned}
$$

Lemma 3.11. Let $k \geq 2$. Consider the binary decomposition

$$
k=\sum_{i=0}^{d} a_{i} 2^{i}, \quad a_{i} \in\{0,1\}, \quad a_{d}=1 .
$$

Let $x \in A_{\infty} \cap B_{k}$. Then there exists $y \in A_{\infty} \cap B_{2^{d}}$ such that $x \Delta_{k} \geq y$ and

$$
\chi_{i}(y)-\chi_{i}(x)=a_{i}+a_{i-1} \sum_{j=i}^{d} a_{j} 2^{j-i}, \quad i=1, \ldots, d .
$$

Proof. Induction by $\nu(k)$ - the number of ones in the binary decomposition of $k$. If $\nu=1$, then $k=2^{d}$ and $a_{0}=\cdots=a_{d-1}=0$, hence, (23) holds for $y=x \Delta_{k}=x \Delta_{2^{d}}$.

Assume that the statement is proved for all $k^{\prime}$ with $\nu\left(k^{\prime}\right)<\nu(k)$ and let us prove it for $k$. Let $2^{e-1}$ be the maximal power of 2 which divides $k$, i.e., $\left(a_{0}, \ldots, a_{d}\right)=$ $\left(0, \ldots, 0,1, a_{e}, \ldots, a_{d}\right)$. Let $k^{\prime}=k-2^{e-1}$. Then $k^{\prime}=\sum a_{i}^{\prime} 2^{i}$ where $\left(a_{0}^{\prime}, \ldots, a_{d}^{\prime}\right)=$ $\left(0, \ldots, 0,0, a_{e}, \ldots, a_{d}\right)$. By Lemma 3.10, there exists $x^{\prime} \in A_{\infty} \cap B_{k^{\prime}}$ such that $x \Delta_{k} \geq x^{\prime} \Delta_{k^{\prime}}$ and $\chi\left(x^{\prime}\right)-\chi(x)=\left(n_{1}, \ldots, n_{d}\right)=(0, \ldots, 0,1, p, 0, \ldots, 0)$ where $p=k^{\prime} / 2^{e}=\sum_{j=e}^{d} a_{j} 2^{j-e}, n_{e-1}=1$, and $n_{e}=p$.

Since $\nu\left(k^{\prime}\right)=\nu(k)-1$, there exists $y \in A_{\infty} \cap B_{2^{d}}$ such that $x^{\prime} \Delta_{k} \geq y$ and (23) holds with $x$ and $a_{i}$ replaced by $x^{\prime}$ and $a_{i}^{\prime}$. Hence,

$$
\begin{aligned}
\chi_{i}(y)-\chi_{i}(x) & =\left(\chi_{i}\left(x^{\prime}\right)-\chi_{i}(x)\right)+\left(\chi_{i}(y)-\chi_{i}\left(x^{\prime}\right)\right)=n_{i}+a_{i}^{\prime}+a_{i-1}^{\prime} \sum_{j=i}^{d} a_{j}^{\prime} 2^{j-i} \\
& = \begin{cases}0+a_{i}+a_{i-1}\left(a_{i}+2 a_{i+1}+\cdots+2^{d-i} a_{d}\right), & i \geq e+1, \\
p+1+0, & i=e, \\
1+0+0, & i=e-1, \\
0+0+0, & i \leq e-2 .\end{cases}
\end{aligned}
$$

This is equal to the right hand side of (23) in all the four cases.

We define arithmetic functions $f(k), g(k)$ via the binary decomposition $(22)$ :

$$
f(k)=\sum_{i=0}^{d} a_{i}+\sum_{0 \leq i<j \leq d} a_{i} a_{j} 2^{j-i-1}, \quad g(k)=a_{d-1}-1+\sum_{i=2}^{d-1} a_{i}\left(1-a_{i-1}\right) .
$$


Corollary 3.12. Let $k$ be as Lemma 3.11. Then there exists $y \in A_{\infty} \cap B_{2^{d}}$, $\chi(y)=\left(n_{1}, \ldots, n_{d}\right)$, such that $\Delta_{k} \geq y$ and

$$
\begin{gathered}
\left(1-(-1)^{n_{i}}\right) / 2=a_{i}\left(1-a_{i-1}\right), \quad i=1, \ldots, d, \\
S_{1} n_{1}+\cdots+S_{d} n_{d}=\left(k^{2}-f(k)\right) / 3 .
\end{gathered}
$$

Proof. By (23) we have $n_{i}=a_{i}+a_{i-1}\left(a_{i}+2 a_{i+1}+\ldots\right) \equiv a_{i}\left(1-a_{i-1}\right) \bmod 2$ and

$$
\begin{aligned}
3 \sum_{i=1}^{d} S_{i} \chi_{i}(y) & =\sum_{i=1}^{d}\left(4^{i}-1\right)\left(a_{i}+a_{i-1} \sum_{j=i}^{d} a_{j} 2^{j-i}\right) \\
& =\sum_{i=0}^{d} a_{i}\left(4^{i}-1\right)+\sum_{i=1}^{d}\left(4^{i}-1\right) a_{i-1} \sum_{j=i}^{d} a_{j} 2^{j-i} \\
& =\sum_{i=0}^{d} a_{i} 4^{i}-\sum_{i=0}^{d} a_{i}+\sum_{0 \leq i<j \leq d} a_{i} a_{j}\left(4^{i+1}-1\right) 2^{j-i-1} \\
& =\sum_{i=0}^{d} a_{i}^{2} 4^{i}+2 \sum_{0 \leq i<j \leq d} a_{i} a_{j} 2^{i+j}-f(k)=k^{2}-f(k) .
\end{aligned}
$$

Theorem 3.13. Let $k \geq 2, n \geq 1$. Let $f$ and $g$ be as in (22), (24). We set $\varepsilon=\left(1-(-1)^{n}\right) / 2, d=\left[\log _{2} n\right]$. Then $\sigma_{1}^{-N} \Delta_{k}^{n}$ is quasipositive for

$$
N=\frac{n\left(k^{2}-f(k)\right)}{3}-2 d+1-\varepsilon g(k)+\left[\frac{n}{4}\right] \max (0, f(k)-g(2 k)-2 d-1) .
$$

Proof. Let $E=f(k)-g(2 k)-2 d-1$. If $E \leq 0$, then the result follows immediately from Corollaries 3.8 and 3.12. Consider the case $E>0$. Let $q=[n / 4], r=n-4 q$. We set $x=\sigma_{1}^{-N_{1}} \Delta_{k}^{r}, y=\sigma_{1}^{-N_{2}} \Delta_{2 k}$, and $z=\sigma_{1}^{-N_{2}} \Delta_{k}^{4}$ where $N_{1}=r\left(k^{2}-f(k)\right) / 3-$ $2 d+1-\varepsilon g(k)$ and $N_{2}=\left((2 k)^{2}-f(2 k)\right) / 3-2 d-1-g(2 k)$. By Corollaries 3.8 and 3.12 , we have $x \geq 1$ and $y \geq 1$. Combining $y \geq 1$ with Corollary 3.4, we obtain $z \geq 1$. Since $f(2 k)=f(k)$, we have $N=N_{1}+q N_{2}$. Thus, $\sigma_{1}^{-N}=x z^{q} \geq 1$.

Proposition 3.14. a). We have $1 \leq f(k) \leq k$ for any $k$. Moreover, $f(k)=k$ iff $k=2^{d+1}-1$ and $f(k)=1$ iff $k=2^{d}$ for some $d \geq 0$.

b). We have $k-f(k)-3 g(2 k) \geq 0$. The equality is attained iff either $k=2^{d+2}-1$ or $k=2^{d+3}-2^{d}-1$ for some $d \geq 0$.

Proof. a).

$$
k-f(k)=\sum_{j=0}^{d} a_{j}\left(2^{j}-1-\sum_{i=0}^{j-1} a_{i} 2^{j-i-1}\right) \geq \sum_{j=0}^{d} a_{j}\left(2^{j}-1-\sum_{i=0}^{j-1} 2^{j-i-1}\right)=0
$$

and we have the equality iff $k=2^{d}-1$. It is evident that $f(k)=1$ iff $k=2^{d}$.

b). Exercise.

Corollary 3.15. a). If $N \leq \frac{2}{3}\left(k^{2}-k\right)-2\left[\log _{2} k\right]+1$, then $\sigma_{1}^{-N} \Delta_{k}^{2}$ is quasipositive. b). If $N \leq \frac{4}{3} k^{2}-\frac{1}{3} k-2\left[\log _{2} k\right]-1$, then $\sigma_{1}^{-N} \Delta_{2 k}$ is quasipositive. 


\section{Curves with a DeEP nest And With many innermost ovals}

\subsection{Real pseudoholomorphic curves.}

Let $A$ be a real curve on $\mathbb{R P}^{2}$. We say that the depth of an oval of $\mathbb{R} A$ is $q$ if it is surrounded by $q$ ovals. Degtyarev, Itenberg, and Kharlamov [7] ask: how many ovals of the depth $k-2$ a curve of degree $2 k$ may have? Note, that $k-2$ is the maximal possible depth of ovals of a non-hyperbolic curve (a curve of degree $2 k$ is called hyperbolic if it has $k$ nested ovals and hence, by Bezout's theorem, it cannot have more ovals). This question appears from the study of the number of components of an intersection of three real quadrics in higher dimensional spaces (see details in [7]).

Let us denote the number of ovals of depth $q$ of a curve $A$ by $l_{q}=l_{q}(A)$. The improved Petrovsky inequality implies $l_{k-2} \leq \frac{3}{2} k^{2}+O(k)$. On the other hand, Hilbert's construction provides curves with $l_{k-2} \geq k^{2}+O(k)$. We improve this lower bound up to $9 / 8 k^{2}$ for algebraic curves (see Proposition 4.3). The results of $\S 3$ (see Theorem 3.13 and Corollary $3.15(\mathrm{~b})$ ) provide a lower bound of the form $4 / 3 k^{2}+O(k)$ for real pseudoholomorphic curves because of the following fact.

Proposition 4.1. The braid $\sigma_{1}^{-N} \Delta_{2 k}$ is quasipositive if and only if there exists a real pseudoholomorphic curve $A$ in $\mathbb{R P}^{2}$ of degree $2 k$ such that $l_{k-2}(A)=N$.

Proof. According to $[22 ; \S 2.3]$, the fiberwise arrangement $\left[\supset_{1} o_{1}^{N-1} \subset_{1}\right]$ is realizable by a real pseudoholomorphic curve of degree $2 k$ if and only if the braid $x=\sigma_{1}^{-N} \Delta_{2 k}$ is quasipositive. Thus, the quasipositivity of $x$ implies the existence of a curve with $l_{k-2}=N$.

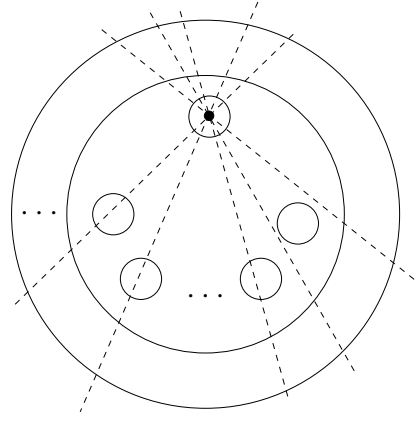

FiguRE 9.1

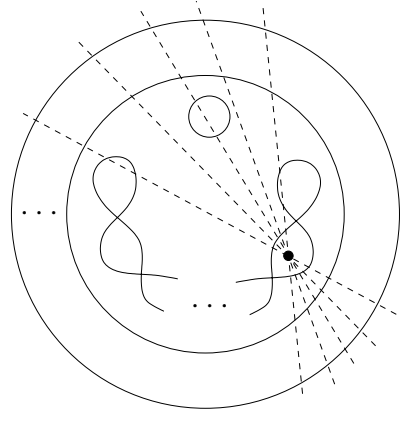

FiguRE 9.2

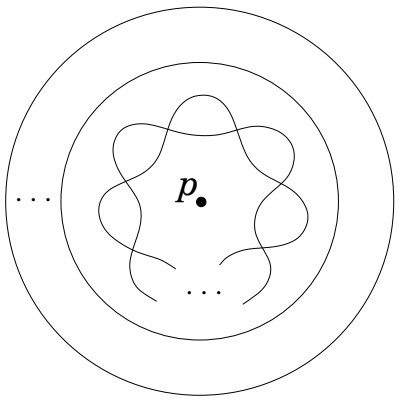

FiguRE 9.3

Suppose that there exists a pseudoholomorphic curve $A$ of degree $2 k$ with $l_{k-2}=$ $N$. Let $v_{1}, \ldots, v_{N}$ be the innermost ovals (i.e., the ovals of depth $k-2$ ). If some arrangement of embedded circles in $\mathbb{R P}^{2}$ is realizable by a real pseudoholomorphic curve and we erase an empty oval, then the new arrangement is also realizable by a real pseudoholomorphic curve. Thus, without loss of generality we may assume that $A$ realizes the isotopy type $1\langle\ldots 1\langle N\rangle \ldots\rangle$. The arguments from [28] based on auxiliary conics through five innermost ovals prove that $v_{1}, \ldots, v_{N}$ are in a convex position. Thus, choosing a pencil of lines centered at $v_{1}$, we see that $v_{2}, \ldots, v_{N}$ form a single chain (see Figure 9.1), hence they can be replaced by a single branch $B$ which has $N-2$ double points (see Figure 9.2). Choosing a pencil of lines as in Figure 9.2, we attach $B$ to $v_{1}$ as in Figure 9.3. The braid corresponding to the arrangement of the obtained curve with respect to the pencil of lines centered at $p$ (see Figure 9.3) is a conjugate of $\sigma_{1}^{-N} \Delta_{2 k}$. 
Corollary 4.2. For any integer $k \geq 2$, there exists a real pseudoholomorphic curve $A$ on $\mathbb{R P}^{2}$ of degree $2 k$ such that $l_{k-2}(A) \geq\left(4 k^{2}-f(k)\right) / 3-2\left[\log _{2} k\right]-1-g(2 k)$ where $f, g$ are as in $(24)$, in particular, $l_{k-2}(A) \geq 4 / 3 k^{2}-1 / 3 k-2\left[\log _{2} k\right]-1$.

\subsection{Real algebraic curves.}

Proposition 4.3. For any $k=4 p$ there exists a real algebraic curve of degree $2 k$ in $\mathbb{R P}^{2}$ such that $l_{k-2}=18 p^{2}-2 p=9 / 8 k^{2}-1 / 2 k$.

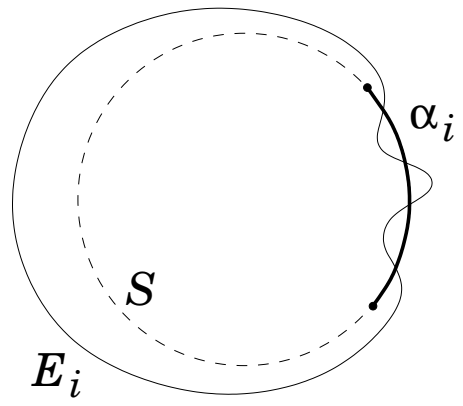

FiguRE 10.1

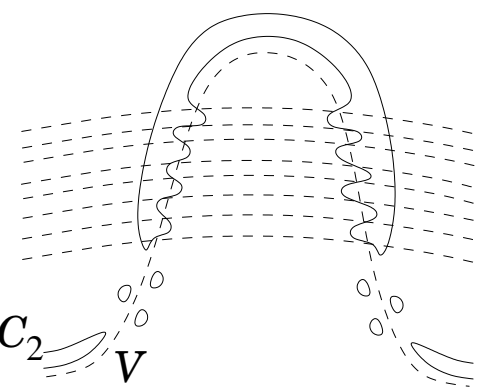

FIGURE 10.4

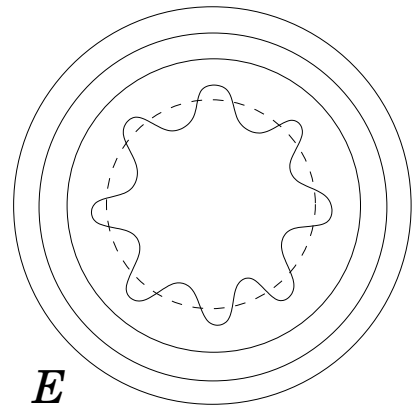

FIGURE 10.2

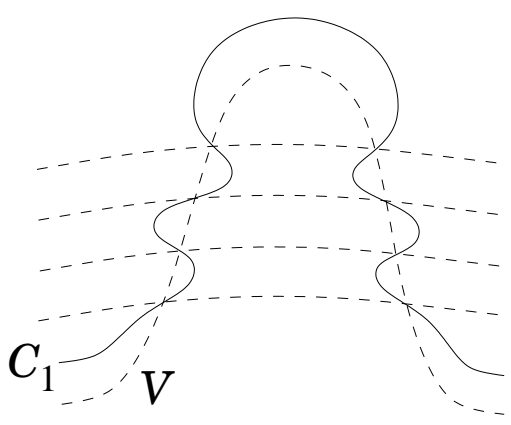

FigURE 10.3

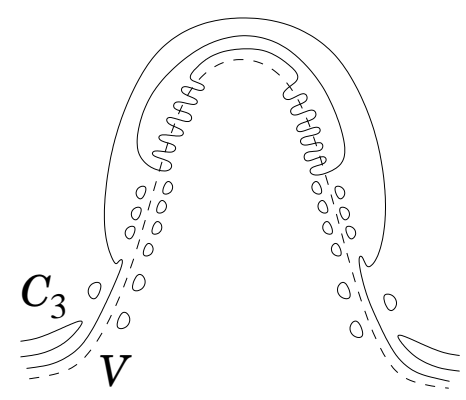

FIGURE 10.5

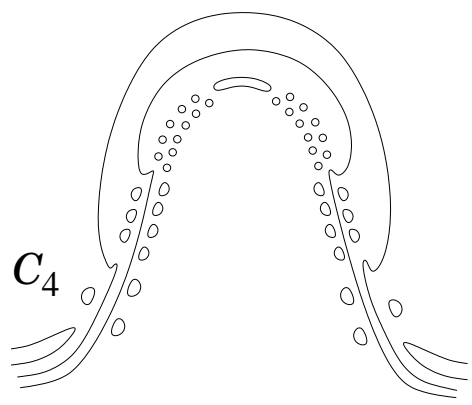

FIGURE 10.6

Proof. We fix an affine chart $\mathbb{R}^{2}$ on $\mathbb{R P}^{2}$. Let $S$ be the unit circle and let $\alpha_{1}, \ldots, \alpha_{p}$ be disjoint arcs of $S$. Let $E_{1}, \ldots, E_{p}$ be ellipses such that $E_{i}$ is arranged on $\mathbb{R}^{2}$ with respect to $S$ and $\alpha_{i}$ as in Figure 10.1. Then $E_{1} \cup \cdots \cup E_{p}$ can be perturbed into a curve $E$ of degree $2 p$ consisting of a single nest of the depth $p$ (i.e., a hyperbolic curve), and the innermost oval $V$ of $E$ intersects $S$ in $k$ points which lye on $S$ in the same order as on $V$ (see Figure 10.2). Let $S_{\nu, 1}, \ldots, S_{\nu, \nu p}, \nu=1, \ldots, 4$, be concentric copies of $S$ of increasing radii $\left(r_{1,1}<\cdots<r_{1, p}<r_{2,1}<\cdots<r_{2,2 p}<r_{3,1}<\ldots\right)$ each of whom intersects $V$ at $k$ points. Let

$$
C_{0}=1, \quad C_{\nu}=E C_{\nu-1}+\varepsilon_{\nu} \prod_{i=1}^{\nu p} S_{\nu, i}, \quad \nu=1, \ldots, 4, \quad 0<\left|\varepsilon_{4}\right| \ll \cdots \ll\left|\varepsilon_{1}\right| \ll 1
$$

(see Figure 10.3 - 10.6; we use the same notation for a curve and its defining polynomial). Then $C_{4}$ is the required curve. 


\section{On $A_{N}$ Singularity of a Plane Curve of a given Degree}

Easy to see that the existence of a pseudoholomorphic curve of degree $m$ which has a singular point of the type $A_{n}$ is equivalent to the quasipositivity of the braid $\sigma_{1}^{-(n+1)} \Delta_{m}^{2}$. Thus, Theorem 3.13 admits also the following interpretation.

Proposition 5.1. For any $m$, there exists a pseudoholomorphic curve $C_{m}$ in $\mathbb{C P}^{2}$ of degree $m$ with a singularity of the type $A_{n}$ with $n=2 / 3\left(m^{2}-m\right)-2\left[\log _{2} k\right]$. Thus, $\lim _{m \rightarrow \infty} 2 n / m^{2}=4 / 3$.

The question what is the maximal $n=N(m)$ such that there exists an algebraic curve of degree $m$ with an $A_{n}$ singularity was studied by several authors. Let $\alpha=\lim \sup 2 N(m) / m^{2}$. Signature estimates for the double covering yield $\alpha \leq 3 / 2$ (see [14]). An evident example $\left(y+x^{k}\right)^{2}-y^{2 k}=0$ yields $m=2 k$ and $n=2 k^{2}-1$, so, $\alpha \geq 1$.

In a generic family of curves, the condition to have an $A_{n}$ singularity defines a stratum of codimension $n$. Thus the so-called expected dimension of the variety of curves of degree $m$ with a singularity $A_{n}$ is equal to $m^{2} / 2-n+O(m)$, i.e., $\alpha>1$ is "unexpected" from this point of view. Nevertheless, this is so. A series of examples providing $\alpha \geq 15 / 14$ was constructed by Gusein-Zade and Nekhoroshev in [14]. Cassou-Nogues and Luengo [4] improved this estimate up to $\alpha \geq 8-4 \sqrt{3}$. Here we show that $\alpha \geq 7 / 6$. This follows from the following evident observation.

Proposition 5.2. Let $F(X, Y)$ be a polynomial whose Newton polygon is contained in the triangle with vertices $(0,0),(a c, 0)$, and $(0, b c)$. Suppose that $F=0$ has a singularity $A_{k-1}$ at the origin, and $\operatorname{ord}_{0} F(0, Y)=2$. Then, for any $p \geq b / a$, the curve $F\left(X^{p b}, Y^{p a}+X\right)=0$ has a singularity $A_{n}$ for $n=a b k p^{2}-1$ and its degree is $m=a b c p$ and hence, $\alpha \geq \lim _{p \rightarrow \infty}\left(2 n / m^{2}\right)=2 k /\left(a b c^{2}\right)$.

Proof. Indeed, $F_{1}(X, Y)=F\left(X^{p b}, Y\right), F_{2}(X, Y)=F_{1}(X, Y+X)$, and $F_{3}(X, Y)=$ $F_{2}\left(X, Y^{p a}\right)$ have singularities $A_{b k p-1}, A_{b k p-1}$, and $A_{a b k p^{2}-1}$ respectively.

If we apply Proposition 5.2 to a sextic curve in $\mathbb{P}^{2}$ which has an $A_{19}$ singularity $(a=b=1, c=6, k=20)$ then we obtain $\alpha \geq 10 / 9$. The existence of such a curve follows from the theory of K3 surfaces (see, e.g., [36]); an explicit equation is given in $[1, \S 5]$.

If we apply Proposition 5.2 to $a=2, b=1, c=4, k=18$, then we obtain $\alpha \geq 9 / 8$. The existence of polynomials realizing this case can be proven using K3 surfaces (Alexander Degtyarev; private communication). Also, they can be written down explicitely:

$$
\begin{aligned}
\left(x^{3}\right. & \left.+45 x^{4}+y-2787 x^{2} y+60192 y^{2}\right)^{2} \\
& +12\left(x^{8}+(1-87 x) x^{5} y-(42-2943 x) x^{3} y^{2}+(288-36288 x) x y^{3}+66816 y^{4}\right)
\end{aligned}
$$

or $\left(x^{3}+y-5 x^{2} y\right)^{2}-4\left(2 x^{8}+2 x^{5} y+9 x^{4} y^{2}+3 x y^{3}+y^{4}\right)$ (the latter polynomial was found by Ignacio Luengo), To determine the singularity type at the origin, it is enough to compute the multiplicity at $x=0$ of the discriminant with respect to $y$. Here is the corresponding maple code for the second polynomial: 
f : $=\left(\mathrm{x}^{\wedge} 3+\mathrm{y}-5 * \mathrm{x}^{\wedge} 2 * \mathrm{y}\right) \wedge 2-4 *\left(2 * \mathrm{x}^{\wedge} 8+2 * \mathrm{x}^{\wedge} 5 * \mathrm{y}+9 * \mathrm{x}^{\wedge} 4 * \mathrm{y}^{\wedge} 2+3 * \mathrm{x} * \mathrm{y}^{\wedge} 3+\mathrm{y} \wedge 4\right)$;

factor (discrim(f,y));

Finally, if we apply Proposition 5.2 to the case $a=3, b=c=2, k=14$, then we obtain $\alpha \geq 7 / 6$. This case is realizable by the polynomial (also found by Ignacio Luengo)

$$
\begin{aligned}
\left(x^{2}-53 x^{3}\right. & \left.+y-60 x y-\frac{2160}{7} y^{2}\right)^{2} \\
& +\frac{4}{7}\left(5 x^{6}+8 x^{4} y+3 x^{2} y^{2}+41 x^{3} y^{2}+27 x y^{3}+\frac{486}{7} y^{4}\right) .
\end{aligned}
$$

\section{OdD DEGREE CURVES WITH MANY NESTS}

\subsection{Construction of real algebraic $M$-curves of degree $4 d+1$ with 4 nests of depth $d$.}

Let $C$ be a nonsingular real pseudoholomorphic curve of an odd degree $m=2 k+1$ in $\mathbb{R P}^{2}$. We say that an oval of $C$ is even (resp. odd) if it is surrounded by an even (resp. odd) number of other ovals. Let us denote the number of even (resp. odd) ovals by $p$ (resp. by $n$ ). In a joint note with Oleg Viro [32] we proved the following result.

Theorem 6.1. If $k=2 d$ (i.e., $m=4 d+1$ ) and $C$ has 4 disjoint nests of depth $d$, then:

(i) If $C$ is an $M$-curve, then $p-n \equiv k^{2}+k \bmod 8$ (Gudkov-Rohlin congruence);

(ii) If $C$ is an $(M-1)$-curve, then $p-n \pm 1 \equiv k^{2}+k \bmod 8$ (KharlamovGudkov-Krakhnov congruence);

(iii) If $C$ is an $(M-2)$-curve and $p-n+4 \equiv k^{2}+k \bmod 8$, then $C$ is of the type I (Kharlamov congruence);

(iv) If $C$ is of the type I, then $p-n \equiv k^{2}+k \bmod 4$ (Arnold congruence).

This is the first result of this kind for curves of odd degree. If $d=1$, it is trivial. If $d=2$, it was conjectured by Korchagin because he constructed $M$-curves of degree 9 with 4 nests and observed the congruence mod 8. However, starting with $d=3$, curves satisfying the hypothesis of Theorem 6.1 have not been known.

In this section we show the "non-emptiness" of Theorem 6.1 for any $d$ for real algebraic curves.

Proposition 6.2. For any integer $d \geq 1$, there exist a real algebraic $M$-curve of degree $m=4 d+1$ which has 4 disjoint nests of depth $d$. This curve realizes the isotopy type

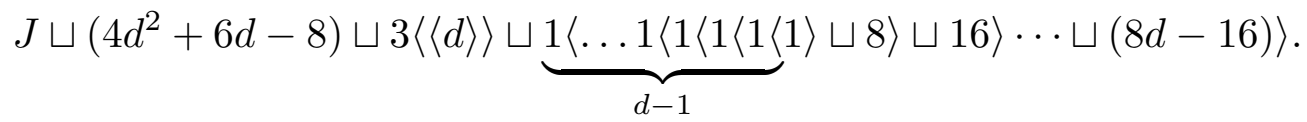

The notation $3\langle\langle d\rangle\rangle$ is explained in $\S 1$.

Proof. The result immediately follows from the following statement $\left(\mathcal{H}_{d}\right)$ which we shall prove by induction:

$\left(\mathcal{H}_{d}\right)$. If $d \geq 1$, then for any $n>0$ there exists a mutual arrangement of an $M$ quartic $Q$, an $M$-curve $C_{d}$ of degree $m=4 d+1$, and $n$ lines $L_{1}, \ldots, L_{n}$ satisfying the following conditions:

(i) the curve $C_{d}$ belongs to the isotopy type (25); 
(ii) each oval of $Q$ (we denote them by $V_{0}, \ldots, V_{3}$ ) surrounds a nest of $C_{d}$ of depth $d$; the nests surrounded by $V_{1}, V_{2}, V_{3}$ are simple;

(iii) one exterior empty oval of $C_{d}$ (let us denote it by $v$ ) intersects $V_{0}$ at $4 m$ distinct points which all lye on $V_{0}$ in the same order as on $v$; so, (Int $\left.V_{0}\right) \backslash$ (Int $v$ ) is a disjoint union of $2 m$ open disks (digons) which we denote by $D_{1}, \ldots, D_{2 m}$

(iv) $C_{d} \cap D_{i}=\varnothing$ for $i>1$ and $C_{d} \cap D_{1}$ has the isotopy type $(8 d-8) \sqcup S_{d}$ where $S_{d}$ stands for the final part of the expression (25) starting with " $1<\ldots$ ";

$(v)$ all the other exterior empty ovals are outside of all the ovals of $Q$;

(vi) there exist arcs $\alpha_{1} \subset \cdots \subset \alpha_{n} \subset V_{0} \cap D_{m+1}$ such that for any $i=1, \ldots, n$, the line $L_{i}$ intersects $Q$ at 4 distinct points which lye on $\alpha_{i} \backslash \alpha_{i-1}$, two points on each connected component of $\alpha_{i} \backslash \alpha_{i-1}$ (here we assume that $\alpha_{0}=\varnothing$ );

Given a line $L$, we shall denote by $L^{k}(\varepsilon)$ a union of $k$ generic lines depending on a real parameter $\varepsilon$ such that each line tends to $L$ as $\varepsilon \rightarrow 0$. We shall use the same notation for a curve and a polynomial which defines it. A notation $0 \ll \cdots \ll$ $\varepsilon_{2} \ll \varepsilon_{1} \ll 1$ means that we choose a small parameter $\varepsilon_{1}$, then we choose $\varepsilon_{2}$ which is small with respect to $\varepsilon_{1}$, and so on.

Let us prove $\left(\mathcal{H}_{1}\right)$. Let $E$ be a conic and let $p_{1}, q_{1}, p_{2}, q_{2}, \ldots, p_{n+3}, q_{n+3}$ be points lying on $E$ in this cyclic order. Let $L_{i}$ be the line $\left(p_{i} q_{i}\right)$ and let us set $Q=E^{2}+\varepsilon_{2} L_{n+3}^{4}\left(\varepsilon_{1}\right)$ and $C_{1}=Q L_{n+2}+\varepsilon_{4} L_{n+1}^{5}\left(\varepsilon_{3}\right)$ where $0 \ll \varepsilon_{4} \ll \cdots \ll \varepsilon_{1} \ll 1$. Then $Q, C_{1}$, and $L_{1}, \ldots, L_{n}$ satisfy $(i)-(v i)_{d=1}$ for a suitable choice of signs of the equations (see Figure 11).
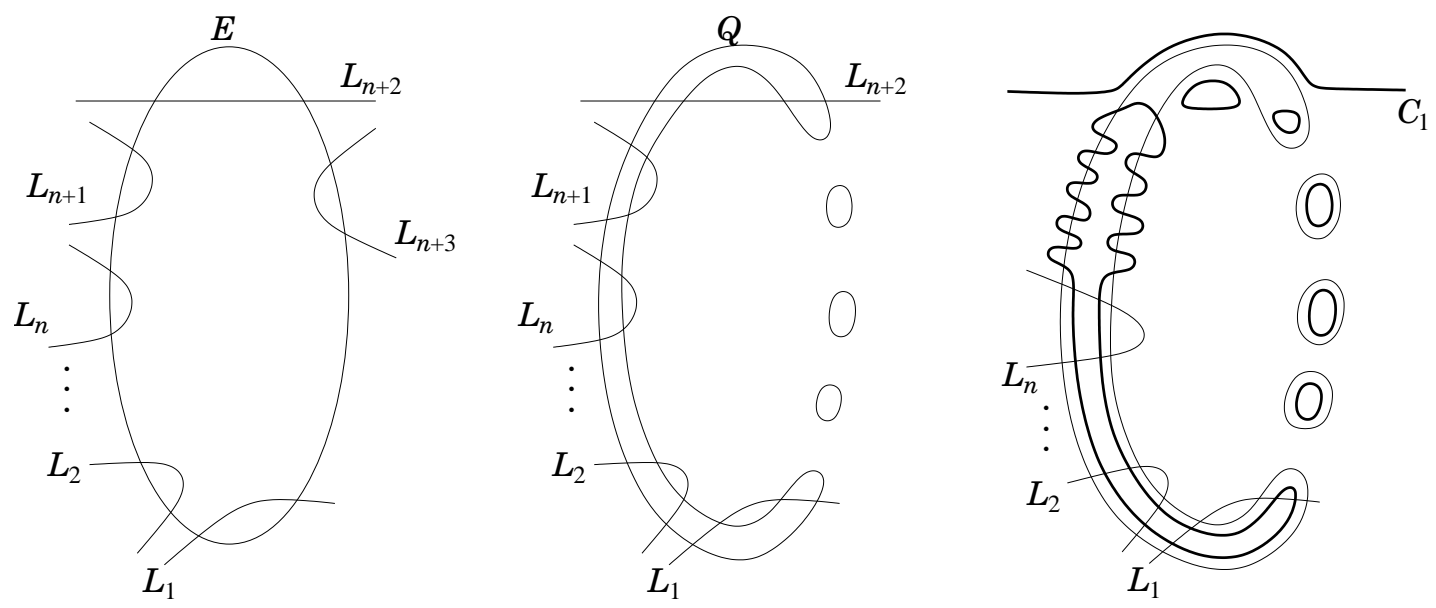

FiguRE 11

Now let us assume that $\left(\mathcal{H}_{d}\right)$ is true and let us prove $\left(\mathcal{H}_{d+1}\right)$. Let $Q, C_{d}$, and $L_{1}, \ldots, L_{n+1}$ satisfy $(i)-(v i)$ with $n+1$ instead of $n$ and let us set $C_{d+1}=$ $Q C_{d}+\delta L_{n+1}^{4 d+5}(\varepsilon)$ with $0 \ll \delta \ll \varepsilon \ll 1$ (see Figure 12).

Remark. For the curve in Proposition 6.2, it is easy to check that $p-n=k^{2}+k$. Indeed, one sees in Figure 12 that $p_{d+1}=n_{d}+4 d^{2}+14 d+6$ and $n_{d+1}=p_{d}-4 d^{2}+2 d$, hence $\left(p_{d+1}-n_{d+1}\right)=-\left(p_{d}-n_{d}\right)+8 d^{2}+12 d+6$, i.e. the quantities $p_{d}-n_{d}$ and $k^{2}+k=(2 d)^{2}+2 d$ satisfy the same recurrent relation. This gives another proof that the right hand side of the congruences in Theorem 6.1 is correctly computed (it 

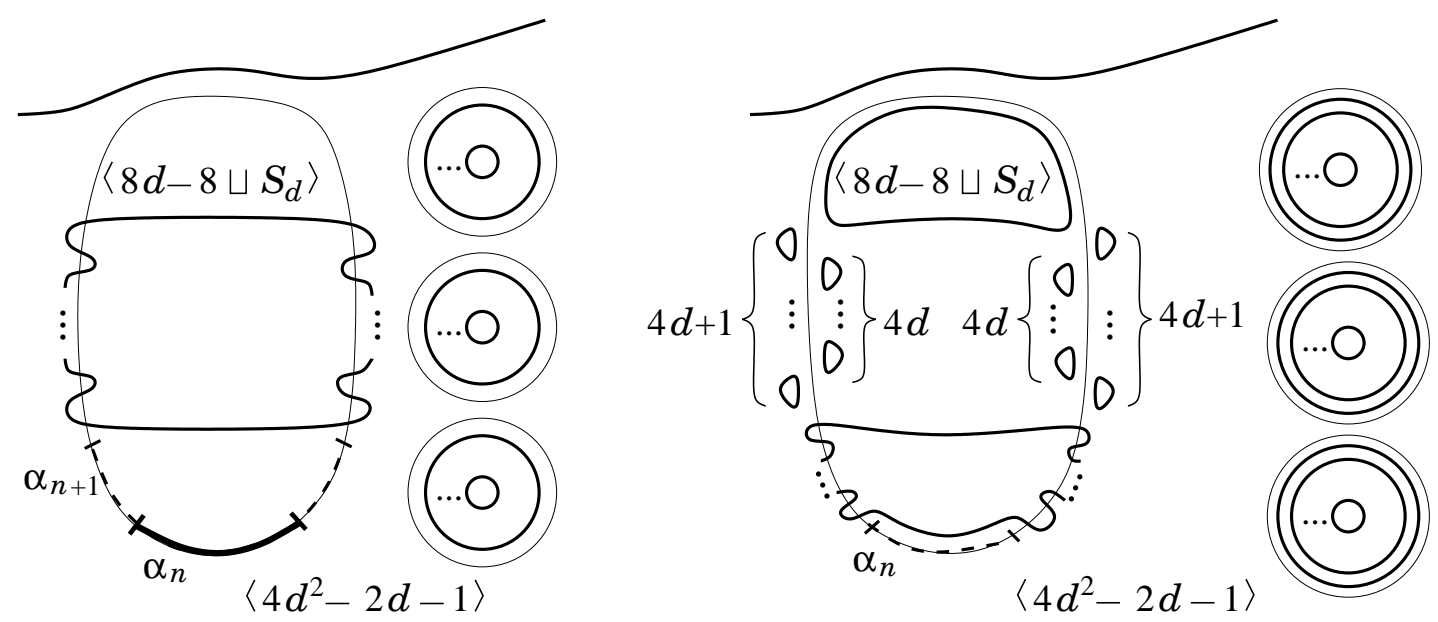

Induction step: $1\left\langle 8 d-8 \sqcup S_{d}\right\rangle=S_{d+1}$;

$\left(4 d^{2}-2 d-1\right)+(8 d+2)=4(d+1)^{2}-2(d+1)-1$.

FIGURE 12

was computed in [32] via Brown-van der Blij invariant of Viro-Kharlamov quadratic form defined in [33]).

\subsection{On $M_{d}$-curves of degree $2 t d+1$.}

Let $A$ be a real algebraic (or real pseudoholomorphic) curve on $\mathbb{R P}^{2}$ of degree $m=2 k+1$ and $k=t d$. Recall that the depth of an oval is the number of ovals which surround it. Let $V$ be an oval of $A$. We say that $V$ is a $d$-oval of $A$ if the depth of $V$ is a multiple of $d$ (maybe, zero) and $V$ is the outermost oval of a nest of depth at least $d$ (i.e., there is at least $d-1$ nested ovals inside $V$ ). We say that $A$ is an $M_{d}$-curve if it is an $M$-curve of degree $m$ and the number of its $d$-ovals is at least $2 t^{2}-3 t+2$.

For example, the curves discussed in $\S 6.1$ are $M_{d}$-curves of degree $4 d+1$ (i.e., $t=2)$.

Proposition 6.3. (a). For any integers $t \geq 2$ and $d \geq 1$ there exist real pseudoholomorphic $M_{d}$-curves of degree $m=2 t d+1$.

(b). For any integer $t \geq 2$ there exist real algebraic $M_{2}$-curves of degree $4 t+1$. In particular:

(c). For any integer $t \geq 2$ there exists a real algebraic $M$-curve of degree $m=$ $4 t+1$ realizing the isotopy type $J \sqcup g_{2 t}\langle 1\rangle \sqcup 1\langle t-1\rangle \sqcup\left(4 t^{2}+3 t-2\right)$ where $g_{2 t}=$ $(t-1)(2 t-1)$ is the genus of a curve of degree $2 t$. So, this curve has as many nests as the number of ovals of an $M$-curve of degree $2 t$.

Proof. (a). Let $B$ be a real algebraic $M$-curve of degree $2 t$ and a line $L$ satisfying the following conditions:

(i) an oval $V$ of $B$ has $2 t$ intersections with $L$ placed on $V$ in the same order as on $L$;

(ii) $B \backslash V \subset E$ where $E$ is the component of $\mathbb{R P}^{2} \backslash(V \cup L)$ whose closure is non-orientable.

Such curve can be easily obtained by Harnack's method (see also the proof of (b)). We construct curves $C_{e}$ of degrees $m_{e}=2 t e+1, e=0,1,2, \ldots$ recursively (see Figure 13). We set $C_{0}=L$ and we define $C_{e+1}$ as a small perturbation of $C_{e} \cup B$ 


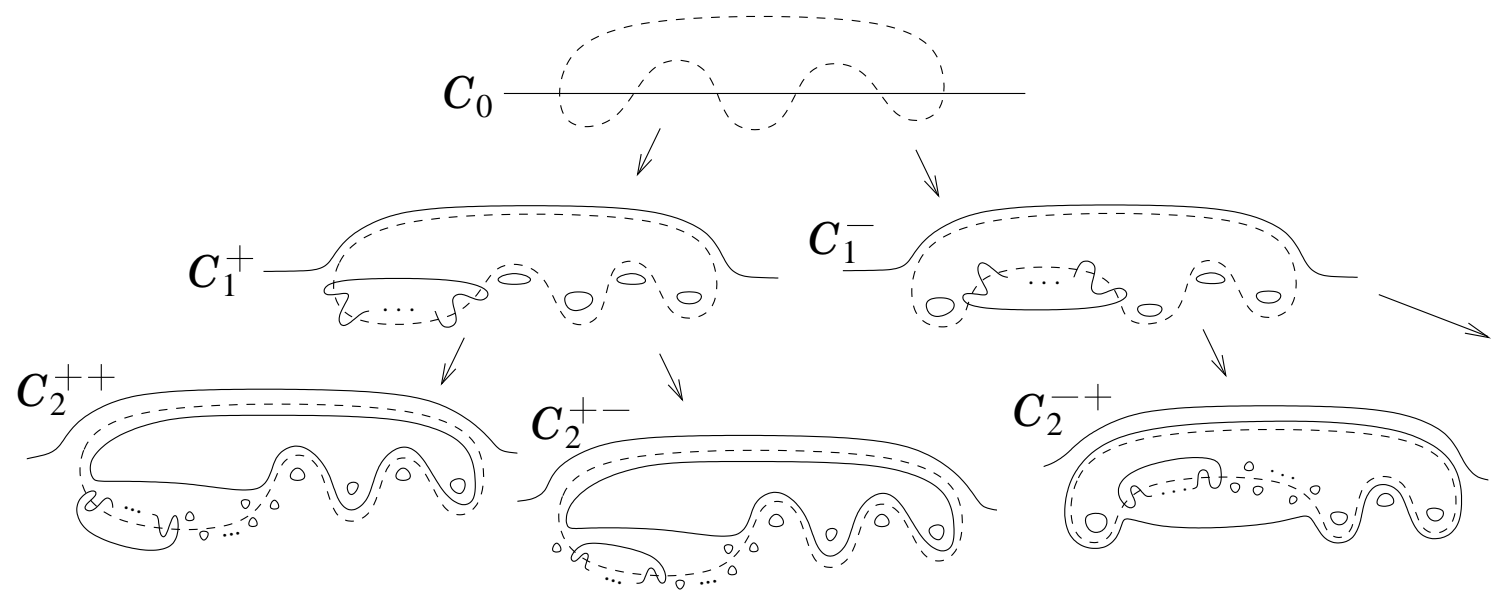

FIGURE 13

such that $C_{e+1}$ meets $B$ at $2 t m_{e}$ points all lying on an arc of $B$ bounding a digon between $B$ and $C_{e}$.

(b). For some curves $B$, the second step of the above construction can be realized in the class of algebraic curves. Suppose that $B$ and $L$ satisfy the conditions $(i)-(i i)$ and, moreover, $V$ and $L$ are arranged with respect to another line $L^{\prime}$ as it is shown in Figure 14. Then we obtain the isotopy type

$$
J \sqcup(a+t-1) \sqcup 1\langle t-1\rangle \sqcup S^{2}
$$

where $a=2 t(2 t+1)-1$ and $S$ is the isotopy type of $B \backslash V$ (see Figure 14).

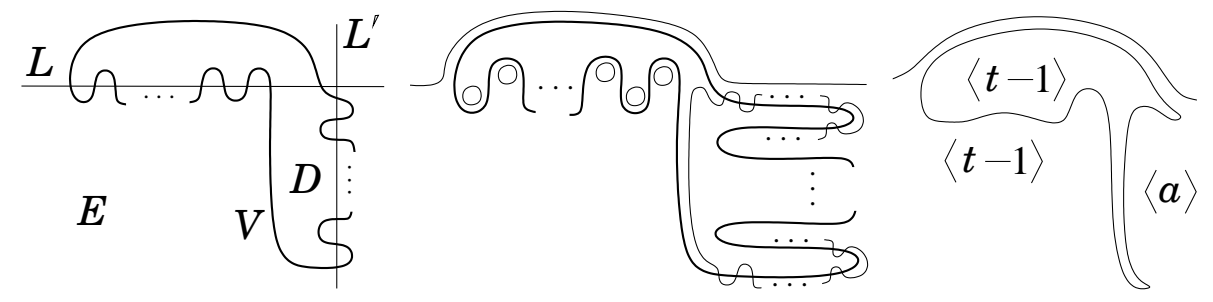

FIGURE 14

To construct the required arrangement of $B, L$, and $L^{\prime}$, we can start with a Harnack curve of degree $2 t-2$ and proceed as it is shown in Figure 15. Here $g_{t}=(t-1)(t-2) / 2$ and $g_{t-1}=(t-2)(t-3) / 2$.
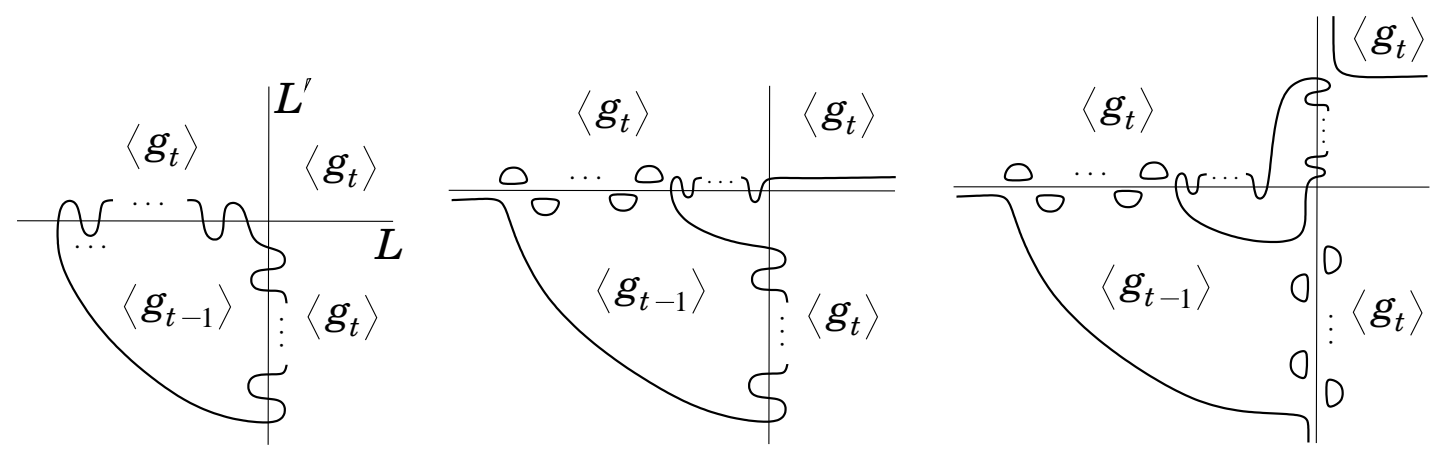

FIGURE 15 
The construction of $\left(B, L, L^{\prime}\right)$ can be interpreted as Viro patchworking according to the Haas' zone decomposition (see [15]) of the triangle $O X Y$ into two triangles and one quadrangle $O P Y, X Y Q$, and $X P Y Q$ (see Figure 16.1) where $O=(0,0)$, $X=(2 t, 0), Y=(0,2 t), P=(1,0)$, and $Q=(1,1)$. This means that we choose any primitive triangulation which contains the edges $X Q, Q Y, Y P$ and we define the sign distribution $\delta:(O X Y) \cap \mathbb{Z}^{2} \rightarrow\{ \pm 1\}$,

$$
\delta(x, y)= \begin{cases}(-1)^{(x+1)(y+1)}, & y>0 \\ -1, & y=0\end{cases}
$$

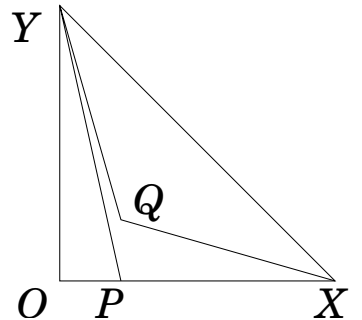

FIGURE 16.1

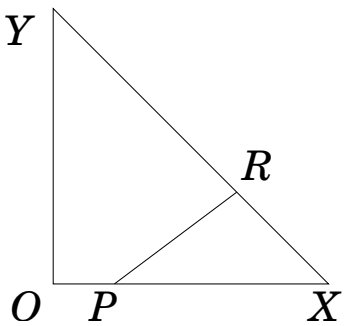

FiguRE 16.2

(c) Let $B$ be the $M$-curve of degree $2 t$ patchworked according to the Haas' zone decomposition of $O X Y$ obtained by cutting it along the segment $P R$ where $O$, $X, Y, P$ are as above and $R=(2 t-2,2)$ (see Figure 16.2). This means that we choose any primitive triangulation which contains the edge $P R$ and we define the sign distribution $\delta:(O X Y) \cap \mathbb{Z}^{2} \rightarrow\{ \pm 1\}$,

$$
\delta(x, y)= \begin{cases}(-1)^{x y}, & (x . y) \in O P R Y, \text { i.e., }(2 t-3) y \geq 2(x-1) \\ (-1)^{(x+1) y}, & (x, y) \in X P R, \text { i.e., }(2 t-3) y \leq 2(x-1),\end{cases}
$$

Then $B$ has an oval $V$ which is arranged with respect to the lines $L$ and $L^{\prime}$ (the axes $O x$ and $O y$ respectively) as in Figure 14, but all other ovals of $B$ are empty. Moreover, $(t-1)(t-2) / 2$ empty ovals are in the domain $D$ and the other empty ovals are in the domain $E$. The rest of the construction is shown in Figure 14.

Remarks. 1. Let $p$ and $n$ be the number of positive and negative ovals of a curve $C_{d}$ constructed in the proof of Proposition 6.3(a). It is easy to prove by induction that

$$
p-n= \begin{cases}2 t\left( \pm m_{1} \pm m_{3} \pm \cdots \pm m_{d-1}\right), & d \text { is even } \\ 2 t\left(1 \pm m_{2} \pm m_{4} \pm \cdots \pm m_{d-1}\right)+p_{B}-n_{B}-2, & d \text { is odd }\end{cases}
$$

where $m_{e}=2 t e+1, p_{B}$ (resp. $n_{B}$ ) is the number of positive (resp. negative) ovals of $B$, and the choice of the signs is illustrated in Figure 13. Thus it follows from Gudkov-Rohlin congruence that for any choice of $B$ satisfying $(i)$ and (ii), we have

$$
p-n \equiv\left\{\begin{array}{lll}
k^{2}+k & \bmod 8 . & \text { if } t \equiv d \equiv 0 \bmod 2, \\
k^{2}+k+t-2 & \bmod 8, & \text { if } t \equiv d+1 \equiv 0 \bmod 2, \\
k^{2}+k & \bmod 4, & \text { if } t+1 \equiv d \equiv 0 \bmod 2, \\
k^{2}+k+t-2 & \bmod 4, & \text { if } t \equiv d \equiv 1 \bmod 2
\end{array}\right.
$$


where $k=t d$ (so, $\left.\operatorname{deg} C_{d}=2 k+1\right)$. All values of $p-n$ satisfying these congruences are attained for pseudoholomorphic curves.

2. The algebraic curves constructed in the proof of Proposition 6.3(b,c) satisfy the congruence $p-n \equiv k^{2}+k \bmod 8$. The first pseudoholomorphic curve constructed in Proposition 6.3(a) which does not satisfy this congruence is the curve of degree $13(t=3, d=2)$ of the isotopy type $J \sqcup 1 \sqcup 1\langle 44\rangle \sqcup 8\langle 1\rangle \sqcup 1\langle 1\langle 1\langle 1\rangle\rangle\rangle$ (the curve $C_{2}^{-+}$in Figure 13 if Harnack's sextic is chosen for $B$ ). It is interesting to study if this curve is algebraically realizable.

\section{7. $M$-CURVES OF DEGREE 9 WITH A SINGLE EXTERIOR OVAL}

Theorem 7.1. (a). There exist real algebraic curves of degree 9 realizing the isotopy types

$$
J \sqcup 1\langle 2 a \sqcup 1\langle 26-2 a\rangle\rangle, \quad 2 \leq a \leq 11 .
$$

(b). The isotopy type $J \sqcup 1\langle 24 \sqcup 1\langle 2\rangle\rangle$ is unrealizable by real pseudoholomorphic (in particular, by real algebraic) curves of degree 9.

Combined with the result of S. Fiedler-LeTouzé [12], Theorem 7.1 implies that among the isotopy types of the form $J \sqcup 1\langle b \sqcup 1\langle 26-b\rangle\rangle$, only the isotopy types in list (26) are realizable by curves of degree 9 .

Following [12; Definition 1], we say that a curve of degree 9 has an $O_{1}$-jump if it has six ovals arranged with respect to some line as in Figure 17. Theorem 7.1(b) follows immediately from [12; Theorem 2(2)] combined with the following fact:

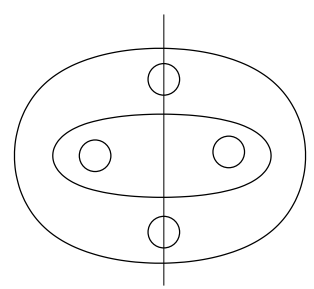

FiguRE 17. $O_{1}$-jump

Theorem 7.2. Let $A$ be an $M$-curve of degree 9 which realizes the isotopy type $J \sqcup 1\langle\beta \sqcup 1\langle\gamma\rangle\rangle$ with $\beta+\gamma=26$. Then $A$ has an $O_{1}$-jump.

Theorem 7.1(a) is proven in $\S 7.1$; Theorem 7.2 is proven in $\S 7.2$.

Recall that an oval of a real algebraic plane curve is called exterior if it is not surrounded by another oval. We say that $A$ is a one exterior oval curve (OEO curve) if it has exactly one exterior oval. Note that OEO $M$-curves of degree greater than three have not been known. It is evident that OEO $M$-curves do not exist in degree 4 and 5 . Petrovsky inequality excludes OEO $M$-curves of degree 6. Viro [28] (resp. Shustin [26]) excluded OEO $M$-curves of degree 7 (resp. 8). Using theta-characteristics (the idea applied later in [7]), Kharlamov excluded OEO $M$ curves of odd degree of a very special form $J \sqcup 1\langle n\rangle$ (unfortunately, his proof still is not written). However, OEO $M$-curves of degree 9 do exist by Theorem 7.1(a).

It seems that OEO $M$-curves of even degree greater that 2 do not exist. Note, that Hilbert's construction provides OEO $(M-r)$-curves of any even degree $\geq 6$ for any $r \geq 1$. 


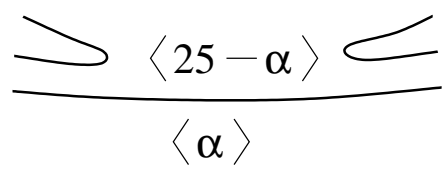

Figure 18. $\alpha \in\{4,8,12,16,20\}$

\subsection{Construction.}

Lemma 7.3. For any $\alpha \in\{4,8,12,16,20\}$ and for any distinct real numbers $\lambda_{1}, \lambda_{2}, \lambda_{3}$, there exists a polynomial $g(x, y)=\sum_{i+9 j \leq 27} g_{i j} x^{i} y^{j}$ such that the affine curve $g(x, y)=0$ is as in Figure 18 and $g^{\Gamma}=\left(y-\lambda_{1} x^{9}\right)\left(y-\lambda_{2} x^{9}\right)\left(y-\lambda_{3} x^{9}\right)$ where $g^{\Gamma}$ denotes the truncation of $g$ to the edge $\Gamma=[(27,0),(0,3)]$ of the Newton polygon, i.e. $g^{\Gamma}=\sum_{i+9 j=27} g_{i j} x^{i} y^{j}$

Proof. Follows easily from the results of [29].

Proof of Theorem 7.1(a). All curves (26) are realizable as perturbations of the singular curve $F_{3}\left(F_{3}^{2}+c F_{2}^{3}\right)=0$ where $F_{3}=0$ is an $M$-cubic and $F_{2}=0$ is a conic which has the maximal tangency with $F_{3}=0$ at a point $p$ lying on the oval $O_{3}$ of the curve $F_{3}=0$.

Let $F_{2}(X, Y)=Y-X^{2}, F_{3}(X, Y)=\left(Y-X^{2}\right)(1+3 Y)+2 Y^{3}, F_{6}=F_{3}^{2}+c F_{2}^{3}$, $0<c \ll 1$, and $F_{9}=F_{6} F_{3}$. Let $C_{k}$ be the curve $F_{k}=0, k=2,3,6,9$. Then $C_{2}$ has the tangency of order 6 at the origin with $C_{3}$ and the mutual arrangement of $C_{2}$ and $C_{3}$ on $\mathbb{R}^{2}$ is as in Figure 19.1. Hence the arrangement of $C_{9}$ on $\mathbb{R P}^{2}$ is as in Figure 19.2. The curve $C_{9}$ has three smooth real local branches at the origin (two branches of $C_{6}$ and one of $C_{3}$ ) with pairwise tangencies of order 9 .

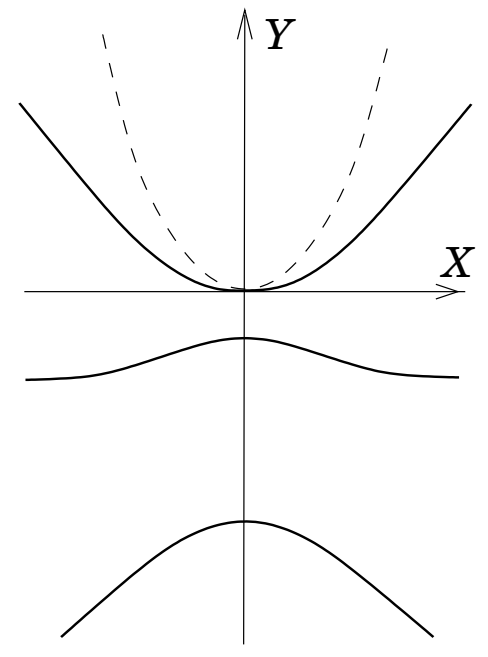

FiguRE 19.1

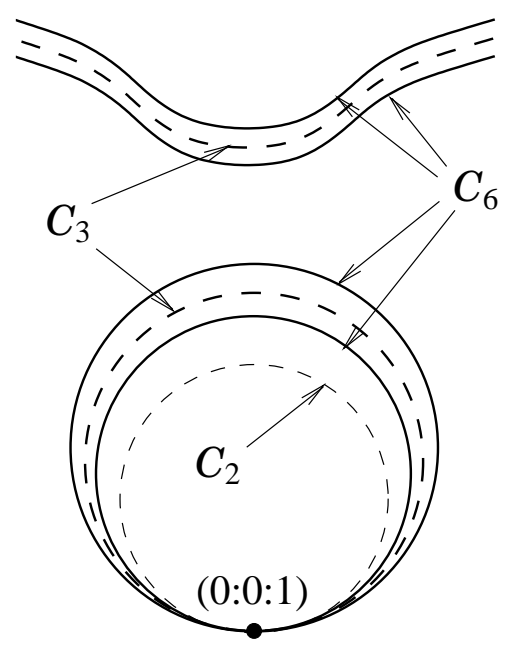

FiguRE 19.2

We introduce local coordinates $(x, y)$ at the origin $X=x, Y=y+\gamma(x), \gamma(x)=$ $x^{2}-2 x^{6}+6 x^{8}$. Let $f_{k}(x, y)=F_{k}(x, y+\gamma(x)), k=2,3,6,9$, i.e., $f_{k}$ is $F_{k}$ rewritten in the coordinates $(x, y)$. Then $f_{9}$ has the form $\sum_{i+9 j \geq 27} a_{i j} x^{i} y^{j}$ and $f_{9}^{\Gamma}=y\left(y^{2}-\right.$ $\left.8 c x^{18}\right)$ where $f_{9}^{\Gamma}$ is the truncation of $f_{9}$ to $\Gamma$, i.e., $f_{9}^{\Gamma}=\sum_{i+9 j=27} a_{i j} x^{i} y^{j}$. Here is the Mathematica code that checks it: 
$\mathrm{F} 2=\mathrm{Y}-\mathrm{X} \wedge 2 ; \mathrm{F} 3=\mathrm{F} 2(1+3 \mathrm{Y})+2 \mathrm{Y}^{\wedge} 3 ; \mathrm{F} 6=\mathrm{F} 3 \wedge 2+\mathrm{C} * \mathrm{~F} 2 \wedge 3 ; \mathrm{F} 9=\mathrm{F} 3 * \mathrm{~F} 6 ;$

$\mathrm{su}=\left\{\mathrm{X}->\mathrm{X}, \mathrm{Y}->\mathrm{y}+\mathrm{x}^{\wedge} 2-2 \mathrm{x}^{\wedge} 6+6 \mathrm{x}^{\wedge} 8\right\} ; \mathrm{f} 9=\mathrm{Expand}[\mathrm{F} 9 / / . \mathrm{su}]$;

Table [Series [Coefficient $[f 9, y, j],\{x, 0,27-9 j\}],\{j, 0,3\}]$

We perturb the singularity of $C_{9}$ at the origin using the straightforward approach from [5]. Let $g(x, y)$ be as in Lemma 7.3 where we set $g^{\Gamma}=f_{9}^{\Gamma}$. We have $g_{18,1}=$ $a_{18,1}=-8 c \neq 0$, hence shifting if necessarily the $x$-coordinate, we may assume that $g_{17,1}=0$.

Let $\tilde{F}(X, Y)=\sum_{i+j \leq 9} B_{i j} X^{i} Y^{j}$ be a polynomial with indeterminate coefficients. We set $\tilde{f}(x, y)=\tilde{F}(x, y+\gamma(x))=\sum_{i, j} b_{i j} x^{i} y^{j}$. Then $b_{i j}$ 's are linear functions of $B_{i j}$ 's. Let $\varphi(i, j)=27-i-9 j$. Solving a system of linear equations, we find $B_{i j}=B_{i j}(t)$ such that

$$
b_{i j}=g_{i j} t^{\varphi(i, j)} \quad \text { for } \quad i+9 j<27, \quad(i, j) \neq(17,1) .
$$

Substituting the solution into $b_{17,1}$ we see that $b_{17,1}=O\left(t^{2}\right)$ :

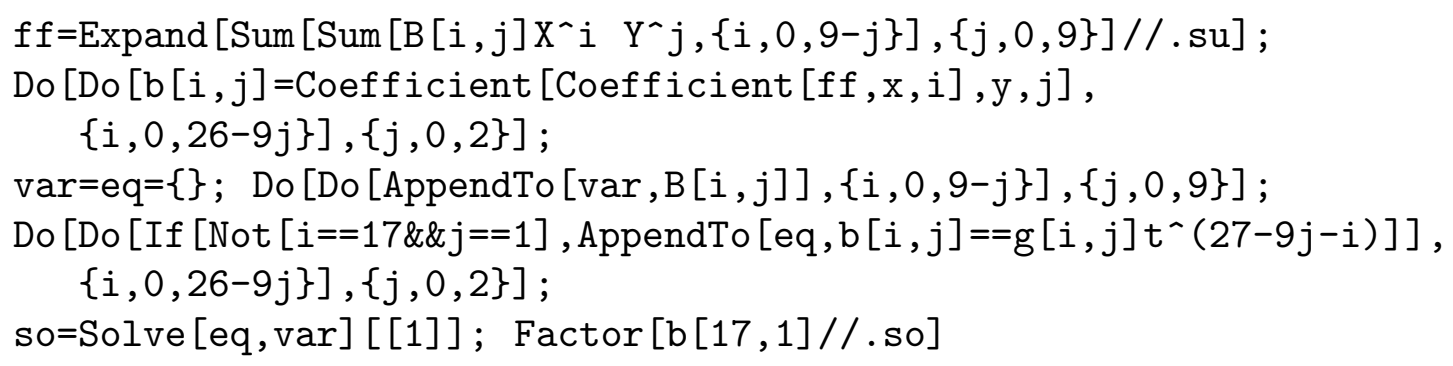

Recall that $g_{17,1}=0$. Thus, for any $(i, j)$ such that $i+9 j<27$, we have $b_{i j}=g_{i j} t^{\varphi(i, j)}+O\left(t^{\varphi(i, j)+1}\right)$. Therefore, the curve $F_{9}(X, Y)+\tilde{F}_{t}(X, Y)=0$ for $0<t \ll c$ is obtained from $C_{9}$ by Viro's patchworking by gluing the pattern in Figure 18 into the singular point of $C_{9}$. We obtain in this way the isotopy types (26) with $a=2,4,6,8,10$. Replacing $g(x, y)$ with $g(x,-y)$, we obtain those with $a=3,5,7,9,11$.

7.2. Restrictions. The main tool used in the proof of Theorem 7.2 is the analog of Murasugi-Tristram inequality for colored signatures obtained in [13,6]. Given a $\mu$-colored oriented link, i.e., an oriented link $L$ in $S^{3}$ with a fixed decomposition $L=L_{1} \sqcup \cdots \sqcup L_{\mu}$ into a disjoint union of sublinks, and a $\mu$-tuple of complex numbers $\omega=\left(\omega_{1}, \ldots, \omega_{\mu}\right),\left|\omega_{i}\right|=1, \omega_{i} \neq 1$, V. Florens [13] defined the isotopy invariants $\omega$-signature $\sigma_{\omega}(L)$ and $\omega$-nullity $\eta_{\omega}(L)$. In [6], D. Cimasoni and V. Florens gave an efficient algorithm for the computation of $\sigma_{\omega}$ and $n_{\omega}$ via a generalized (colored) Seifert surface of $L$. This algorithm was used for the computations in the proof of Theorem 7.2. When $\mu=1$, these invariants specialize to the usual Tristram signature and nullity. They satisfy the following analog of Murasugi-Tristram inequality.

We set $\mathbb{T}_{*}^{1}=\{z \in \mathbb{C} ;|z|=1, z \neq 1\}$ and $\mathbb{T}_{*}^{\mu}=\mathbb{T}_{*}^{1} \times \cdots \times \mathbb{T}_{*}^{1}(\mu$ times $)$.

Theorem 7.4. (See $[6,13])$. Let $F_{1}, \ldots, F_{\mu}$ be disjoint embedded oriented surfaces in the 4-ball $B^{4}$ transversal to the boundary $S^{3}=\partial B^{4}$. Let $F=F_{1} \cup \cdots \cup F_{\mu}$. We consider the colored link $L=L_{1} \sqcup \cdots \sqcup L_{\mu}$ where $L_{i}=\partial F_{i}, i=1, \ldots, \mu$. Then, for any $\omega \in \mathbb{T}_{*}^{\mu}$, we have

$$
\eta_{\omega}(L) \geq\left|\sigma_{\omega}(L)\right|+\chi(F)
$$

where $\chi(F)$ is the Euler characteristic of $F$. 
Remark. In [30, 31], Oleg Viro proposed another approach to define $\eta_{\omega}, \sigma_{\omega}$ and to prove Theorem 7.4. This approach is based on [27].

To reduce the computations, we use the following fact whose proof is very similar to that of [22; Proposition 3.3].

Proposition 7.5. Let $p, q$ be integers such that $0<p<q$ and let $L_{0}$ and $L_{2 q}$ be two $\mu$-colored links represented by braids $b_{0}$ and $b_{2 q}=b_{0} \sigma_{1}^{2 q}$ respectively. Let 1 and 2 be the colors of the first two strings in the part $\sigma_{1}^{2 q}$ of the braid $b_{2 q}$. Let $t=\left(t_{1}, \ldots, t_{\mu}\right) \in \mathbb{T}_{*}^{\mu}$ be such that $t_{1} t_{2}=\exp (2 \pi i p / q)$. Let $t_{j}=\exp \left(2 \pi i \theta_{j}\right)$, $0<\theta_{j}<1, j=1,2$, and $\theta=\theta_{1}+\theta_{2}$. Then $\eta_{t}\left(L_{2 q}\right)=\eta_{t}\left(L_{0}\right)$ and $\sigma_{t}\left(L_{2 q}\right)=$ $\sigma_{t}\left(L_{0}\right)+(q-2 p) \operatorname{sign}(1-\theta)$.

Corollary 7.6. Let $p, q$ be integers such that $0<p<q$. Let $\left\{L_{2 n}\right\}_{n \in \mathbb{Z}}$ be a family of $\mu$-colored links such that $L_{2 n}$ is represented by the braid $b_{2 n}=a_{1} \sigma_{h}^{2 n} a_{2} \sigma_{\ell}^{-2 n} a_{3}$ with some fixed braids $a_{1}, a_{2}, a_{3}$. Let $j$ and $k$ be the colors of the $h$-th and the $(h+1)$-th strings of the part $\sigma_{h}^{2 n}$ of $b_{2 n}$. Suppose that the unordered pair of the colors of the $\ell$-th and the $(\ell+1)$-th strings of the part $\sigma_{\ell}^{-2 n}$ of $b_{2 n}$ is also $\{j, k\}$ (we do not claim that $j \neq k)$. Let $t=\left(t_{1}, \ldots, t_{\mu}\right) \in \mathbb{T}_{*}^{\mu}$ be such that $t_{j} t_{k}=\exp (2 \pi i p / q)$. Then $\eta_{t}\left(L_{2 q}\right)=\eta_{t}\left(L_{0}\right)$ and $\sigma_{t}\left(L_{2 q}\right)=\sigma_{t}\left(L_{0}\right)$.

Proof. If $j=k$, the statement follows from [22; Proposition 3.3]. If $j \neq k$, it follows from Proposition 7.5.

Proof of Theorem 7.2. Suppose that $A$ has no $O_{1}$-jump. Then, applying [22; Corollary 2.3] to a pencil of lines centered at a point inside an empty oval of depth 1 , we may replace the group of the $\gamma$ innermost ovals by a singular branch with $\gamma-1$ double points as shown in Figure 20. It follows from [12; proof of Theorem 2(2)] that if we choose $p$ as in Figure 20, then the fiberwise arrangement of the obtained curve with respect to $\mathcal{L}_{p}$ (the pencil of lines through $p$ ) is $\left[\times_{2}^{\gamma-2} \supset_{2} o_{3}^{\beta_{1}} o_{6}^{\beta_{2}} o_{3}^{\beta_{3}} o_{6}^{\beta_{4}} C_{7} \times_{8}\right]$ for some odd $\beta_{1}, \ldots, \beta_{4}$ such that $\beta_{1}+\cdots+\beta_{4}=\beta$; see $[22 ; \S 2.2]$ for the notation of fiberwise arrangements.

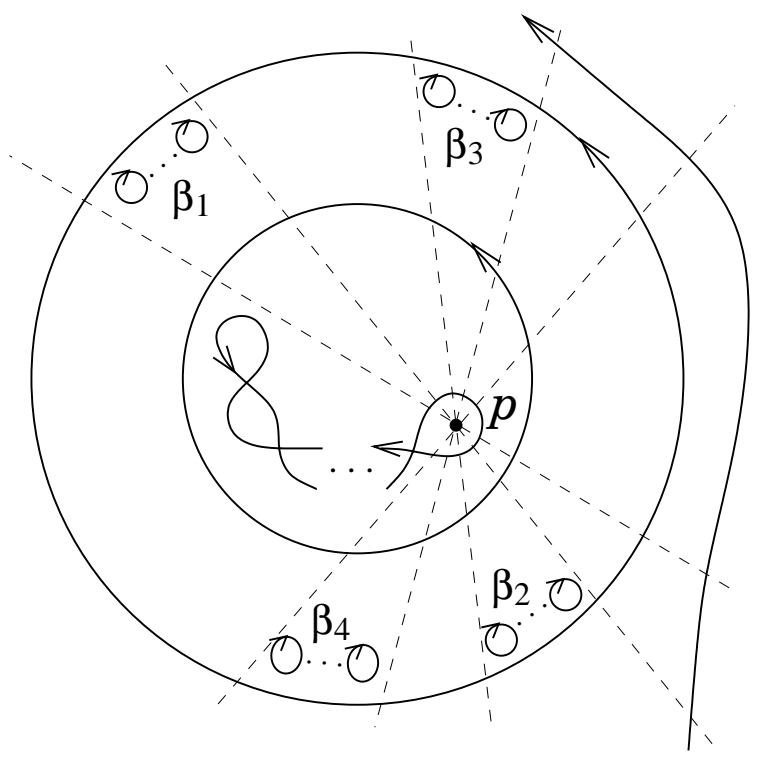

FiguRe 20

Let $b$ be the braid corresponding to $\left(\mathbb{R} A, \mathcal{L}_{p}\right)$. To fix the notation, we reproduce the definition of $b$ from [19]. Let $\pi_{p}: \mathbb{C P}^{2} \backslash p \rightarrow \mathbb{C P}^{1}$ be the linear projection from 
p. We fix complex orientations on $\mathbb{R} A$ and $\mathbb{R P}^{1}$. Let $A \backslash \mathbb{R} A=A_{+} \sqcup A_{-}$and $\mathbb{C P}^{1} \backslash \mathbb{R P}^{1}=\mathbb{C P}_{+}^{1} \sqcup \mathbb{C P}_{-}^{1}$ be the corresponding partitions. Let $H_{+}$be a closed disk in $\mathbb{C P}_{+}^{1}$ containing all nonreal critical values of $\left.\pi_{p}\right|_{A}$. We define $b$ as the closed braid corresponding to the braid monodromy of the curve $A$ along the loop $\partial H_{+}$. We set also $F=\pi_{p}^{-1}\left(H_{+}\right) \cap A, F_{ \pm}=F \cap A_{ \pm}, L=\partial F$, and $L_{ \pm}=\partial F_{ \pm}$. Then $L$ is the braid closure of $b$ in the 3 -sphere $\partial\left(\pi_{p}^{-1}\left(H_{+}\right) \backslash U_{p}\right)$ where $U_{p}$ is a small ball centered at $p$. We have (see $[22 ; \S 2.3]$ )

$$
b=\sigma_{2}^{-\gamma-1} \tau_{2,3} \sigma_{3}^{-\beta_{1}} \tau_{3,6} \sigma_{6}^{-\beta_{2}} \tau_{6,3} \sigma_{3}^{-\beta_{3}} \tau_{3,6} \sigma_{6}^{-\beta_{4}} \tau_{6,7} \sigma_{8}^{-1} \Delta_{9}
$$

where $\tau_{i, j}=\tau_{j, i}^{-1}=\left(\sigma_{i+1}^{-1} \ldots \sigma_{j}^{-1}\right)\left(\sigma_{i} \ldots \sigma_{j-1}\right)$ for $i<j$. It follows from [12] that the complex orientation of $\mathbb{R} A$ is as in Figure 20. Hence, in the braid (28), the strings $1,8,9$ represent $L_{+}$and the strings $2, \ldots, 7$ represent $L_{-}$.

To make the notation coherent with Theorem 7.4 , we set $L_{1}=L_{+}, L_{2}=L_{-}$, $F_{1}=F_{+}, F_{2}=F_{-}$. Riemann-Hurwitz formula for the projection $\left.\pi_{p}\right|_{F}: F \rightarrow H_{+}$ yields $\chi(F)=9-e(b)$ where $e: B_{9} \rightarrow \mathbb{Z}$ is the abelianization homomorphism, i.e., $e(b)$ is the number of branch points of the mapping $\left.\pi_{p}\right|_{F}$. So, we have $\chi(F)=$ $9-10=-1$.

The result follows from the fact that for any choice of four odd numbers $\beta_{1}, \ldots, \beta_{4}$ with $\beta_{1}+\cdots+\beta_{4} \leq 24$, there exist $t=\left(t_{1}, t_{2}\right) \in \mathbb{T}_{*}^{2}$ such that the inequality (27) fails. To reduce the computations, we apply Corollary 7.6. Indeed, suppose that for some $\vec{\beta}^{(0)}=\left(\beta_{1}^{(0)}, \ldots, \beta_{4}^{(0)}\right)$ we find $t$ such that $\operatorname{Arg} t_{1}+\operatorname{Arg} t_{2} \equiv 2 \pi p / q \bmod 2 \pi$ and (27) fails. Then, for any $\vec{\beta}=\left(\beta_{1}, \ldots, \beta_{4}\right)$ such that $\vec{\beta} \equiv \vec{\beta}^{(0)} \bmod 2 q$, the inequality (27) also fails for the same $t$.

By chance, it happens that for any $\vec{\beta}$ there exists $t=\left(t_{1}, t_{2}\right)$ with $t_{1} t_{2}=-1$, so, $q=2$. Thus, it is enough to make the computations, for example, only when each of $\beta_{1}, \ldots, \beta_{4}$ is equal to 1 or 3 . In all these 16 cases, the parameter choice $\left.\left.t_{1}=-1 / t_{2}=\exp \left(2 \pi i \theta_{1}\right), \theta_{1} \in\right] 1 / 6,7 / 40\right]$ provides $\eta_{t}(L)=1,\left|\sigma_{t}(L)\right|=4$, which contradicts (27). When $\gamma \equiv 2 \bmod 4$ (this is enough for Theorem 7.1), one can chose a larger interval $] 1 / 6,3 / 16]$ for $\theta_{1}$. Note that the extremal value $\theta_{1}=1 / 6$ yields $\eta_{t}(L)=2,\left|\sigma_{t}(L)\right|=3$ which does not contradict $(27)$.

\section{REFERENCES}

1. E. Artal, J. Carmona, J. I. Cogolludo, On sextic curves with big Milnor number, in: Trends in Singularities (A. Libgober, M. Tibǎr, eds.), Trends Math., Birkhäuser, Basel, 2002, pp. 1-29.

2. M. Boileau, S. Yu. Orevkov, Quasipositivité d'une courbe analytique dans une boule pseudoconvexe, C. R. Acad. Sci. Paris, Sér. I 332 (2001), 825-830.

3. S. Burckel, The wellordering on positive braids, J. Pure Appl. Algebra 120 (1997), 1-17.

4. Pi. Cassou-Nogues, I. Luengo, On $A_{k}$ singularities on plane curves of fixed degree, Preprint, Oct. 31, 2000.

5. B. Chevallier, Four $M$-curves of degree 8, Funct. Anal. Appl. 36 (2002), 76-78.

6. D. Cimasoni, V. Florens, Generalized Seifert surfaces and signatures of colored links, Trans. Amer. Math. Soc. 360 (2008), 1223-1264.

7. A. Degtyarev, I. Itenberg, V. Kharlamov, On the number of components of a complete intersection of real quadrics, arXiv:0806.4077.

8. P. Dehornoy Braid groups and left distributive operations, Trans. AMS 345 (1994), 115-150.

9. E. A. El-Rifai, H. R. Morton, Algorithms for positive braids, Quart. J. Math. Oxford (2) 45 (1994), 479-497.

10. O. G. Eroshkin, On a topological property of the boundary of an analytic subset of a strictly pseudoconvex domain in $\mathbb{C}^{2}$, Mat. Zametki 49 (1991), no. 5, 149-151 (Russian); English transl. Math. Notes 49 (1991), 546-547. 
11. R. Fenn, M. T. Greene, D. Rolfsen, C. Rourke, B. Wiest, Ordering of the braid groups, Pacific J. Math. 191 (1999), 49-74.

12. S. Fiedler-LeTouzé, $M$-curves of degree 9 with deep nests, J. London Math. Soc, (2) 79 (2009), 649-662.

13. V. Florens, Signatures of colored links with application to real algebraic curves, J. Knot Theory Ramifications 14 (2005), 883-918.

14. S. M. Gusein-Zade, N. N. Nekhoroshev, On $A_{k}$ singularities on plane curves of fixed degree, Funk. Anal. i Prilozh. 34 (2000), no. 3, 69-70 (Russian); English transl., On singularities of type $A_{k}$ on plane curves of a chosen degree, Funct. Anal. and Appl. 34 (2000), 214-215.

15. B. Haas, Real algebraic curves and combinatorial constructions, Ph.D. Thesis, Basel Univ., 1997.

16. V. M. Kharlamov, S. Yu. Orevkov, The number of trees half of whose vertices are leaves and asymptotic enumeration of plane real algebraic curves, J. of Combinatorial Theory, Ser. A 105 (2004), 127-142.

17. R. Laver, Braid group action on left distributive structures and well-ordering in the braid groups, J. Pure Appl. Algebra 108 (1996), 81-98.

18. G. Mikhalkin, Real algebraic curves, the moment map and amoebas, Ann. of Math. (2) 151 (2000), 309-326..

19. S. Yu. Orevkov, Link theory and oval arrangements of real algebraic curves, Topology 38 (1999), 779-810.

20. S. Yu. Orevkov, Strong positivity in the right-invariant order on a braid group and quasipositivity, Mat. Zametki 68 (2000), no. 5, 692-698 (Russian); English transl., Math. Notes 68 (2000), 588-593.

21. S. Yu. Orevkov, Markov moves for quasipositive braids, C. R. Acad. Sci. Paris, Sér. I 331 (2000), 557-562.

22. S. Yu. Orevkov, Classification of flexible $M$-curves of degree 8 up to isotopy, GAFA - Geom. and Funct. Anal. 12 (2002), 723-755.

23. S. Yu. Orevkov, Quasipositivity problem for 3-braids, Turkish J. of Math. 28 (2004), 89-93.

24. S. Yu. Orevkov, V. V. Shevchishin, Markov theorem for transversal links, J. Knot Theory and Ramifications 12 (2003), 905-913.

25. L. Rudolph, Algebraic functions and closed braids, Topology 22 (1983), 191-202.

26. E. I. Shustin, New restrictions on the topology of real curves of degree a multiple of 8 , Izv. AN SSSR (Russian); English transl., Math. USSR-Izvestiya 37 (1991), 421-443.

27. O. Ya. Viro, Signatures of links, Tezisy VII Vsesoyuznoj topologicheskoj konferencii (1977), page 41 (Russian); English transl. http://www.pdmi.ras.ru/olegviro/respapers.html.

28. O. Ya. Viro, Plane real curves of degree 7 and 8: new restrictions, Izv. AN SSSR (Russian); English transl., Math. USSR-Izvestiya 23 (1984), 409-422.

29. O. Ya. Viro, Real algebraic plane curves: constructions with controlled topology, Leningrad J. Math. 1 (1990), 1059-1134.

30. O. Ya. Viro, Acyclicity of circle, twisting-untwisting and their applications, Talk on the Conference "Géométrie et topologie en petite dimension (dédiée au 60ème anniversaire d'Oleg Viro)", CIRM, Luminy, November 17-21, 2008.

31. O. Viro, Twisted acyclicity of a circle and signatures of a link, J. Knot Theory and Ramifications 6 (2009), 729-755.

32. O. Ya. Viro, S. Yu. Orevkov, Congruence modulo 8 for real algebraic curves of degree 9, Uspekhi Mat. Nauk 56:4 (2001), 137-138 (Russian); English transl., Russian Math. Surv. 56 (2001), 770-771.

33. O. Ya. Viro, S. Yu. Orevkov, Congruence modulo 8 for real algebraic curves of degree 9, Extended version. Available on http://picard.ups-tlse.fr/orevkov.

34. B. Wiest, Dehornoy's ordering of the braid groups extends the subword ordering, Pacific J. Math. 191 (1999), 183-188.

35. A. Wiman, Über die reellen Züge der ebenen algebraischen Kurven, Math. Ann. 90 (1923), $222-228$.

36. J. G. Yang, Sextic curves with simple singularities, Tohoku Math J. (2) 48 (1996), $203-227$.

imT, Université Paul Sabatier, 118 route de Narbonne, Toulouse, France

Steklov Mathematical Institute, Gubkina 8, Moscow, Russia

E-mail address: orevkov@math.ups-tlse.fr 